\title{
Hubbard trees
}

by

\section{Alfredo Poirier (Lima)}

\begin{abstract}
We provide a full classification of postcritically finite polynomials as dynamical systems by means of Hubbard trees. The information encoded in these objects is solid enough to allow us recover all the relevant statical and dynamical aspects of the corresponding Julia sets.
\end{abstract}

1. Introduction. In their joint 1984-1985 work, Douady and Hubbard [DH] suggested a combinatorial description for the dynamics of postcritically finite polynomials by means of a tree-like structure, a Hubbard tree (see also [D] ). In this introduction we recall their original construction and isolate the basic features. Then we proceed backwards and impose necessary and sufficient conditions on a dynamical tree for it to become the Hubbard tree of some postcritically finite polynomial. For the sake of completeness we recollect also some elementary, but nevertheless important, properties associated to the Julia set of postcritically finite polynomials.

Given a degree $d \geq 2$ polynomial $f$, we consider its filled Julia set $K(f)$ defined as the set of points whose orbit remains bounded. This set is known to be compact and full (i.e., its complement consists of a unique unbounded component). The behavior under iteration of the critical points dramatically influences the topology of $K(f)$. For example, this set is connected if and only if it traps all critical orbits. We are interested in the special case where the orbit of every critical point is finite, or what is the same, the case when all critical orbits are periodic or eventually periodic. We call such polynomials postcritically finite. For them, the filled Julia set $K(f)$, besides being connected, is also locally connected (cf. [M]).

The boundary $\partial K(f)$ of the filled Julia set, denoted by $J(f)$, is the Julia set and its elements are Julia points. Its complement $F(f)=\mathbb{C}-J(f)$ is the Fatou set. A periodic orbit that contains a critical member is a critical

2010 Mathematics Subject Classification: Primary 37F10; Secondary 37E25.

Key words and phrases: Hubbard tree, holomorphic dynamics, postcritically finite rational maps. 
cycle. In the postcritically finite setting, a periodic orbit belongs to $F(f)$ if and only if it is a critical cycle (for details we refer to [M, Corollary 14.5]).

In this postcritically finite case, the dynamics admits a further decomposition. When restricted to the interior of $K(f)$ (which happens to be nonempty only under the appearance of a critical cycle), the polynomial $f$ maps each bounded Fatou component-always simply connected-onto some other as a branched covering map. Furthermore, all are eventually periodic (see [M, Theorem 16.4]). Each component can be uniformized so that in local charts $f$ reads $z \mapsto z^{m}$ for some $m \geq 1$ (see [M, Theorem 9.1]). More is true. If $U$ is a periodic bounded Fatou component, then the first return map is conjugated to $z \mapsto z^{k}$, this time with $k \geq 2$. In particular, loops of components are in perfect correspondence with critical cycles. Also, in each component there is a unique point which eventually maps to a critical point (precisely the one marked as 0 in local coordinates), referred to in the future as the center.

It is well known (see for instance [DH, Corollary VII.4.2, p. 64]) that given a degree $d \geq 2$ postcritically finite polynomial $f$, for any $z \in K(f)$ the sets $K(f)-\{z\}$ and $J(f)-\{z\}$ consist each of a finite number of connected pieces. In this way, the filled Julia set can be thought of as arranged in a tree like fashion.

To prune this object of inessentials, we pick a finite invariant set $M$ that contains all critical points. Within $K(f)$ we interconnect $M$ by $\operatorname{arcs}$ subject to the extra condition that when a Fatou component is met, then this intersection consists of radial segments in the associated coordinate. Douady and Hubbard proved that this construction defines a finite topological tree $T(M)$ when $M$ together with the intrinsic branching points are considered vertices.

The vertex dynamics is invariant and carries the endpoints of any edge to distinct elements, so that it can be extended to a function from $T(M)$ to itself which is one-to-one on each edge and is isotopic to $f$, the original map. We also keep record of the local degree at every vertex $v$ as $d(v)$. In addition, if three or more edges meet at a vertex, then their cyclic order should be remembered. In other words, we specify how this tree is embedded in the complex plane, again, up to isotopy.

Unfortunately, this data alone is not enough to determine the affine conjugacy class of $f$. For example, the left and right hand sides of Figure 4.6 illustrate Julia sets which are mirror images of each other (hence, are non-equivalent) yet they render the same minimal tree. Douady and Hubbard noticed that if we append enough information to recover the inverse tree - and there are several ways to state this unambiguously - then different postcritically finite polynomials yield different structures. No criterion 
for realization was given at the time. (The only previous partial results in this direction are stated in Lavaurs' thesis $[\mathrm{L}]$.)

To formally deal with this condition we introduce angles around vertices (this again should be credited to [DH, p. 46]). In what follows we measure angles in turns, so that 1 degree is priced $1 / 360$ of a turn. At the center of a component the angle between edges is measured using the local chart. Near Julia vertices, where $m$ components of $K(f)$ intersect, the angle is naturally defined as a multiple of $1 / \mathrm{m}$.

These angles satisfy two obvious conditions. First, they are compatible with the embedding of the tree. Second, they satisfy the identity

$$
\angle_{f(v)}\left(f(e), f\left(e^{\prime}\right)\right) \equiv d(v) \angle_{v}\left(e, e^{\prime}\right)(\bmod 1),
$$

where $d(v)$ is the local degree at $v$, and $e, e^{\prime}$ are edges incident at $v$. When this further structure is provided, we are facing a Hubbard tree, denoted by $H_{f, M}$.

Next we move in reverse: we start with an abstract dynamical tree and we reconstruct the appropriate postcritically finite polynomial.

Abstract Hubbard trees. An angled tree $H$ is a finite simplicial tree together with a function $e, e^{\prime} \mapsto \angle_{v}\left(e, e^{\prime}\right) \in \mathbb{Q} / \mathbb{Z}$ which assigns a rational modulo 1 to each pair of edges $e, e^{\prime}$ incident at a vertex $v$. This angle $\angle_{v}\left(e, e^{\prime}\right)$ is skew symmetric with $\angle_{v}\left(e, e^{\prime}\right)=0$ if and only if $e=e^{\prime}$, and is subject to $\angle_{v}\left(e, e^{\prime \prime}\right)=\angle_{v}\left(e, e^{\prime}\right)+\angle_{v}\left(e^{\prime}, e^{\prime \prime}\right)$ whenever applicable. This mechanism determines a preferred isotopy class of embeddings of $H$ into $\mathbb{C}$.

Let $V$ be the set of vertices in $H$. We specify a vertex dynamics $f: V \rightarrow V$ subject to $f(v) \neq f\left(v^{\prime}\right)$ whenever $v, v^{\prime}$ are contiguous through an edge $e$. We also consider a local degree $d: V \rightarrow\{1,2, \ldots\}$. We require the total degree $d_{H}=1+\sum_{v \in V}(d(v)-1)$ to be greater than 1 . By definition a vertex is critical if $d(v)>1$ and noncritical otherwise. The critical set is, thus, nonvoid.

We require that $f$ and the degree $d$ be related as follows. Extend $f$ to a map $f: H \rightarrow H$ that carries each edge homeomorphically onto the shortest path joining the images of its endpoints. We then require $\angle_{f(v)}\left(f(e), f\left(e^{\prime}\right)\right)=$ $d(v) \angle_{v}\left(e, e^{\prime}\right)$ whenever $e, e^{\prime}$ are incident at $v$ (so that $f(e), f\left(e^{\prime}\right)$ intersect at $f(v)$, where the angle is measured).

A vertex $v$ is periodic if $f^{\circ k}(v)=v$ for some $k \geq 1$. The orbit of a periodic critical point is a critical cycle. A vertex is of Fatou type if it eventually maps to a critical cycle; else it is of Julia type or a Julia vertex.

The distance $\operatorname{dist}_{H}\left(v, v^{\prime}\right)$ between vertices in $H$ counts the number of edges in the shortest path joining $v$ to $v^{\prime}$. We call $H$ expanding if for every edge $e$ whose endpoints $v, v^{\prime}$ are Julia vertices there is $n \geq 1$ for which we have $\operatorname{dist}_{H}\left(f^{\circ n}(v), f^{\circ n}\left(v^{\prime}\right)\right)>1$. 
Angles at Julia vertices are rather artificial, so it is better to normalize them. If $m$ edges $e_{1}, \ldots, e_{m}$ meet at a periodic Julia vertex $v$, then each $\angle_{v}\left(e_{i}, e_{j}\right)$ should be a multiple of $1 / m$. (Therefore, angles around a periodic Julia vertex convey no information beyond the cyclic order of the edges.) An angled tree that satisfies this condition around each periodic Julia vertex is said to be normalized.

By an abstract Hubbard tree - or simply a Hubbard tree - we mean a normalized angled tree that obeys the expanding condition.

The basic existence and uniqueness theorem is stated now as follows. Its proof is the sole purpose of this paper.

TheOREM 1.1. A normalized dynamical angled tree can be realized as the tree associated to a postcritically finite polynomial if and only if it is expanding. In other words, all Hubbard trees - and only them - can be realized. Such a realization is unique up to affine conjugation.

A point $z \in J(f)$ is an end (or a terminal point) if there is just one external ray landing at $z$. Else $z$ is an incidence point. For incidence points we specialize between branching (where more than two rays land) and nonbranching. For a postcritically finite polynomial $f$, every branching point is periodic or preperiodic. Also, every periodic incidence point is already present as a vertex in any version $H_{f, M}$. All this is formalized below.

Proposition 1.2. For a postcritically finite polynomial, any (Julia set) branching point is either periodic or preperiodic.

Proposition 1.3. Let $f$ be a postcritically finite polynomial and $z \in$ $J(f)$ a periodic incidence point. For any finite invariant set $M$ that contains all the critical points of $f$ we have $z \in H_{f, M}$. Furthermore, the number of components of $H_{f, M}-\{z\}$ is independent of $M$ and equals the number of components of $J(f)-\{z\}$.

These facts are elementary and are restated as Propositions 3.6 and 3.7, respectively.

A conceptual overview. This paper is organized in three parts. First we study actual Hubbard trees, the ones arising from postcritically finite polynomials. Then we turn our attention to abstract Hubbard trees. Third, and last, we show how to recover concrete postcritically finite polynomials from abstract trees.

In Section 2 we introduce the basics. Even if most of the material is known, from these lines we extract our most valuable asset: the expanding condition stated as Theorem 2.17. In Section 3 we observe how a Hubbard tree grows as we take inverses. Among other details we establish that periodic points where two or more external rays land already appear in minimal 
trees, and therefore in all trees. The transition between the first and the second part is done in Section 4: we exhibit examples of actual trees, and trees that for one reason or another fail to live up to our expectations. Here we also hint the consequences of internal symmetries.

In Section 5 we present an abstract counterpart to the theory. There and in Section 6 we retrace our steps and establish analogues to the key results in Section 2. For practical reasons Section 7 is devoted to the study of the local picture around abstract Fatou points. Section 8 mimics Section 3: we prove that taking inverses is a canonical operation and nothing is risked by doing so. Section 9 again acts as a buffer, this time between the second and the third part. Hubbard trees are supposed to provide a concise picture of the filled Julia set, however, for polynomial dynamics, there is important information hidden in the basin of attraction of $\infty$. In fact, as there are external rays and landing point identifications, we have ahead the task of assigning potential arguments to "accessible" Julia places.

The realization of Hubbard trees relies now on the theory of critical portraits (cf. [P3]). As this is a dual framework, our work from Sections 9 and 13 narrows the gap between the two theories. In Section 10 we construct a formal critical portrait from our data. In Section 11 we promote this portrait to admissible. Finally, in Section 12 we prove that the postcritically finite polynomial associated to this admissible critical portrait takes the original abstract tree as Hubbard tree.

Section 14 includes two appendices. We give necessary and sufficient conditions for a finite cyclic map to become multiplication by an integer. Also, and for the benefit of our readers, we survey the main results behind the theory of critical portraits for postcritically finite polynomials.

2. Regulated trees. We start our work by reviewing some properties of Hubbard trees as originally introduced by Douady and Hubbard in [DH].

We will manipulate a special kind of regulated arcs. These paths are compatible with the dynamics in the sense that their images and preimages under $f$ are piecewise regulated. Once we detect the sole source of conflict in the folding around critical points, we find inside $K(f)$ the regulated hull generated by the critical orbit. When we restrict to this invariant tree, we get a simplified, yet accurate, picture of the dynamics of our postcritically finite polynomial.

Let $f$ be postcritically finite. Any two points in the closure of a bounded Fatou component can be joined by a unique arc formed by at most two radial segments. We call such arcs regulated. The filled Julia set $K(f)$, being connected and locally connected in a compact subset of the plane, is arcwise connected. Hence, given two elements $z_{0}, z_{1}$ in $K(f)$ there exists an arc $\gamma: I=[0,1] \rightarrow K(f)$ with $\gamma(i)=z_{i}$. We will not distinguish between a 
path and its image unless strictly necessary. If the intersection of this arc with the closure of every Fatou component is regulated, we keep the name regulated for the arc. For the theory it is crucial that any two points in the filled Julia set are joined by a unique regulated arc.

Lemma 2.1. Two points in $K(f)$ can be joined by a unique regulated arc.

Proof. To settle existence, we start with an arbitrary arc $\gamma: I \rightarrow K(f)$ and perform a minor surgery without disturbing the endpoints. We enumerate all bounded Fatou components as $U_{1}, U_{2}, \ldots$ If $\gamma$ never touches $\bar{U}_{i}$, where $\bar{U}_{i}$ stands for the closure of $U_{i}$, we forget about $U_{i}$. Else, let $t_{0}$ be the first time when $\gamma\left(t_{0}\right)$ belongs to $\bar{U}_{i}$, and $t_{1}$ the last. If $t_{0}=t_{1}$, we again ignore $U_{i}$. However, if $t_{0}<t_{1}$, we modify $\gamma$ inside $\left[t_{0}, t_{1}\right] \subset[0,1]$ so that from now on it joins $\gamma\left(t_{0}\right)$ to $\gamma\left(t_{1}\right)$ - these points are different since $\gamma$ is an arcin a regulated way. We wind up with a continuous redefinition of $\gamma$. In the limit we are left with a continuous injective function because the diameters of the Fatou components shrink to zero.

To prove uniqueness, suppose $\gamma_{1}, \gamma_{2}: I \rightarrow K(f)$ are different regulated arcs with the same endpoints, so that the set $\gamma_{1}(I) \cup \gamma_{2}(I)$ encloses at least one nonempty connected open set $V$. For this set $V$ the maximum principle guarantees $V \subset K(f)$. Since nontrivial open connected sets of $K(f)$ are subsets of a Fatou component $U$, by construction the portions of $\gamma_{1}, \gamma_{2}$ that surround $V$ lie in $\bar{U}$. This is the desired contradiction because two points in $\bar{U}$ are joined by a unique regulated arc.

In what follows we write $\left[z_{0}, z_{1}\right]$ for the regulated path between $z_{0}, z_{1}$. The following property for regulated arcs is obvious (see also [DH, Chapter 2]).

Lemma 2.2. Any subarc of a regulated arc is regulated. Also, the intersection of two regulated arcs is regulated. In particular, given $z_{1}, z_{2}, z_{3} \in K(f)$ there is a unique $p \in K(f)$ such that $\left[z_{1}, z_{2}\right] \cap\left[z_{2}, z_{3}\right]=\left[z_{2}, p\right]$. In this case we have $z_{2}=p$ if and only if $\left[z_{1}, z_{2}\right] \cup\left[z_{2}, z_{3}\right]$ is a regulated arc.

A subset $X \subset K(f)$ is convex regulated-regulated from now on-if $z_{0}, z_{1} \in X$ implies $\left[z_{0}, z_{1}\right] \subset X$. As the intersection of regulated sets is regulated, we define the regulated hull $[X]$ of $X \subset K(f)$ as the minimal convex regulated subset of $K(f)$ that contains $X$. The next result is valid only because we are working with a special kind of paths.

LEMma 2.3. Two regulated sets with nonempty intersection have regulated union.

Proof. Fix $q$ in the intersection. Any $z_{1}, z_{2}$ (in either set) can be connected to $q$ by definition. By Lemma 2.2 , there is $p$ such that $[p, q]=$ $\left[z_{1}, q\right] \cap\left[z_{2}, q\right]$. Again by Lemma 2.2, the arc $\left[z_{1}, p\right] \cup\left[p, z_{2}\right]$ is regulated. 
We will be manipulating sets generated by a finite number of points, so it is better to have an adequate picture of the topological layout. In the lemma below, notice that by definition every end of $\left[z_{1}, \ldots, z_{n}\right]$ is one of the $z_{i}$. The opposite, however, might fail.

Proposition 2.4. The regulated hull of a finite collection of points is a finite topological tree.

Proof. We proceed by induction. For a single element, there is nothing to prove as a point is a degenerate tree. Suppose now $\left[z_{1}, \ldots, z_{n}\right]$ is a finite topological tree and let $z_{n+1}$ be arbitrary in $K(f)$. Let $p$ be the first point in the arc $\left[z_{n+1}, z_{1}\right]$ that tops the closed set $\left[z_{1}, \ldots, z_{n}\right]$. On the one hand $\left[z_{1}, \ldots, z_{n+1}\right]$ contains $\left[z_{1}, \ldots, z_{n}\right] \cup\left[p, z_{n+1}\right]$ by minimality, while on the other it is contained within by construction. Knowing the sets are equal, the result is established.

The next batch of results allows us to produce new regulated arcs from old ones. Here we use the dynamical stability of the construction.

Lemma 2.5. Let $\left[z_{1}, z_{2}\right]$ be a regulated arc with no critical point of $f$, except, possibly, for the endpoints. Then $f$ is injective when restricted to $\left[z_{1}, z_{2}\right]$, and $f\left(\left[z_{1}, z_{2}\right]\right)=\left[f\left(z_{1}\right), f\left(z_{2}\right)\right]$ is regulated.

Proof. Let $\gamma: I \rightarrow K(f)$ be the injective map that defines the regulated arc $\left[z_{1}, z_{2}\right]$. We will prove that $f \circ \gamma$ is one-to-one. The second part follows easily.

First we suppose that $f \circ \gamma$ is locally one-to-one everywhere. (Local injectivity holds automatically at $t_{0}$ whenever $\gamma\left(t_{0}\right)$ is noncritical.) In that case the set $\Delta=\left\{\left(t_{1}, t_{2}\right): t_{1}<t_{2}\right.$ and $\left.f\left(\gamma\left(t_{1}\right)\right)=f\left(\gamma\left(t_{2}\right)\right)\right\}$ is compact. So, if $\Delta$ were nonempty, we could find $\left(t_{1}, t_{2}\right) \in \Delta$ with $t_{2}-t_{1}>0$ minimal. Then, by definition, for every $s, t$ subject to $0<t-s<t_{2}-t_{1}$ the restriction of $f \circ \gamma$ to $[s, t]$ is a regulated arc. If we take $t$ in $\left(t_{1}, t_{2}\right)$, the restrictions of $f \circ \gamma$ to $\left[t_{1}, t\right]$ and $\left[t, t_{2}\right]$ will give different regulated paths between $f(\gamma(t))$ and $f\left(\gamma\left(t_{1}\right)\right)=f\left(\gamma\left(t_{2}\right)\right)$, contrary to Lemma 2.1.

If we manage to show that $f \circ \gamma$ is also injective at potential critical extremes, then we are done. For the sake of argument assume $\gamma(0)$ to be critical. Since $\gamma$ is injective and $f$ is analytic, the set $\{t \in[0,1]: f(\gamma(t))=$ $f(\gamma(0))\}$ is discrete. Hence, we can take $\epsilon$, with $0<\epsilon<1$, so that $t \in(0, \epsilon)$ implies $f(\gamma(t)) \neq f(\gamma(0))$. If $f \circ \gamma$ were not injective near 0 , then we can find $t_{1}<t_{2}<\epsilon$ with $f\left(\gamma\left(t_{1}\right)\right)=f\left(\gamma\left(t_{2}\right)\right)$. In particular, $t_{1}>0$ and we quickly run into trouble: as there are no critical points in $\left[\gamma\left(t_{1}\right), \gamma\left(t_{2}\right)\right]$, the restriction of $f$ to this regulated arc is injective in light of what was shown in the first paragraph; but it is not.

LEMMA 2.6. Let $\gamma(I)$ be a regulated arc with no critical value of $f$ except maybe for the endpoints. Then any lift of $\gamma(I)$ by $f$ is a regulated arc. 
Proof. Since the image of $\gamma$ restricted to $(0,1)$ contains no critical value, the curve can be lifted in exactly $d=\operatorname{deg}(f)$ ways. All are legal.

The following technical result is key to the invariance of trees (compare Lemma 2.11). Write $\Omega_{f}$ for the critical set of $f$.

Lemma 2.7. For any $z_{0}, z_{1} \in K(f)$ we have

$$
\left[f\left(z_{0}\right), f\left(z_{1}\right)\right] \subset f\left(\left[z_{0}, z_{1}\right]\right) \subset\left[f\left(z_{0}\right), f\left(z_{1}\right), f\left(\Omega_{f}\right)\right] .
$$

Proof. Let $\gamma$ represent $\left[z_{0}, z_{1}\right]$. Let $0 \leq t_{1}<\cdots<t_{k} \leq 1$ be the times when $z_{t_{i}}=\gamma\left(t_{i}\right)$ is critical. Also write $z_{t_{0}}=z_{0}, z_{t_{k+1}}=z_{1}$. Break $\left[z_{0}, z_{1}\right]$ as $\left[z_{t_{0}}, z_{t_{1}}\right] \cup \cdots \cup\left[z_{t_{k}}, z_{t_{k+1}}\right]$. Then Lemma 2.5 yields the equality $f\left(\left[z_{0}, z_{1}\right]\right)=$ $f\left(\left[z_{t_{0}}, z_{t_{1}}\right]\right) \cup \cdots \cup f\left(\left[z_{t_{k}}, z_{t_{k+1}}\right]\right)=\left[f\left(z_{t_{0}}\right), f\left(z_{t_{1}}\right)\right] \cup \cdots \cup\left[f\left(z_{t_{k}}\right), f\left(z_{t_{k+1}}\right)\right]$. This union, however, due to Lemma 2.3 , is a regulated set. As $f\left(z_{0}\right), f\left(z_{1}\right)$ already belong to $f\left(\left[z_{0}, z_{1}\right]\right)$, we should have $\left[f\left(z_{0}\right), f\left(z_{1}\right)\right] \subset f\left(\left[z_{0}, z_{1}\right]\right)$.

For the other inclusion note that by assumption we have $z_{t_{i}} \in\left\{z_{0}, z_{1}, \Omega_{f}\right\}$. Hence $f\left(z_{t_{i}}\right), f\left(z_{t_{i+1}}\right) \in\left[f\left(z_{0}\right), f\left(z_{1}\right), f\left(\Omega_{f}\right)\right]$. This, however, implies we have $\left[f\left(z_{t_{i}}\right), f\left(z_{t_{i+1}}\right)\right] \subset\left[f\left(z_{0}\right), f\left(z_{1}\right), f\left(\Omega_{f}\right)\right]$. Pasting together inclusions along the way we get $f\left(\left[z_{0}, z_{1}\right]\right) \subset\left[f\left(z_{0}\right), f\left(z_{1}\right), f\left(\Omega_{f}\right)\right]$.

COROLlary 2.8. The image of a regulated set is regulated.

Proof. Let $X$ be regulated. For any $z_{1}, z_{2}$ in $X$, we get $\left[f\left(z_{1}\right), f\left(z_{2}\right)\right] \subset$ $f\left(\left[z_{1}, z_{2}\right]\right) \subset f(X)$ from Lemma 2.7 .

As hinted by the proof of Lemma 2.7, our only worry is an eventual folding around a critical point. This is explained next.

Proposition 2.9. For $X \subset K(f)$ we have

$$
[f(X)] \subset f([X]) \subset\left[f\left(X \cup \Omega_{f}\right)\right] .
$$

Proof. From $X \subset[X]$ we get $f(X) \subset f([X])$. As $f([X])$ is regulated, this implies $[f(X)] \subset f([X])$.

Given $f(z) \in f([X])$, take $z_{1}, z_{2} \in X$ so that $z \in\left[z_{1}, z_{2}\right]$. Then Lemma 2.7 shows $f(z) \in f\left(\left[z_{1}, z_{2}\right]\right) \subset\left[f\left(z_{1}\right), f\left(z_{2}\right), f\left(\Omega_{f}\right)\right] \subset\left[f(X) \cup f\left(\Omega_{f}\right)\right]=$ $\left[f\left(X \cup \Omega_{f}\right)\right]$.

Corollary 2.10. If $X$ contains the critical set, then $f([X])=[f(X)]$.

In general, given $X$, we denote by

$$
\mathcal{O}(X)=\left\{f^{\circ n}(x): n \geq 0, x \in X\right\}
$$

its forward orbit.

Let $M$ be a finite invariant set containing $\Omega_{f}$. Write $T(M)$ for the regulated hull $[M]$. The minimal tree $T\left(M_{0}\right)$ is the one generated by $M_{0}=$ $\mathcal{O}\left(\Omega_{f}\right)$, the orbit of the critical set. This last tree is usually referred to in the literature as the Hubbard tree of $f$. 
Lemma 2.11. For any invariant set $M$ containing $\Omega_{f}$ we have

$$
f(T(M))=[f(M)] \subset T(M)=[M] .
$$

Proof. As $M \supset \Omega_{f}$, the first equality is guaranteed by Corollary 2.10. Also, since $M$ is invariant we have $f(M) \subset M \subset[M]=T(M)$, and the inclusion follows.

To understand the dynamics in a Hubbard tree, it is good to detect portions where $f$ is injective. We write $\tilde{T}(M)$ for the family of the closures of the components of $T(M)-\Omega_{f}$. In an informal language, we chop the tree at the critical places.

Proposition 2.12. The map $f$ induces a continuous function from $T(M)$ into itself, where the restriction to every member of $\tilde{T}(M)$ is injective.

Proof. The first part follows from Lemma 2.11 and the second from Lemma 2.5.

Given $z \in T(M)$, the incidence $\nu_{T(M)}(z)$ of $T(M)$ at $z$ is the number of components of $T(M)-\{z\}$. In other words, the value $\nu_{T(M)}(z)$ counts how many branches of $T(M)$ stem from $z$. This number might be different from the number of connected components of $K(f)-\{z\}$, the incidence number at $z$ for $f$.

A point $z \in T(M)$ is a branching point of $T(M)$ if $\nu_{T(M)}(z)>2$, and an end if $\nu_{T(M)}(z)=1$. The preferred set of $T(M)$ is $V_{T(M)}=M \cup\{z \in T(M)$ : $\left.\nu_{T(M)}(z)>2\right\}$. As there are only a finite number of vertices in those trees, the collection $V_{T(M)}$ is finite.

LEMma 2.13. The set $V_{T(M)}$ is invariant and its regulated hull is equal to $T(M)$.

Proof. As $M$ is invariant and contains $\Omega_{f}$, we limit ourselves to check if the image of a noncritical branching point is also branching. This, however, is trivial: from $\nu_{T(M)}(z)>2$ and $\operatorname{deg}_{z} f=1$ we jump to $\nu_{T(M)}(f(z))>2$ because $f$ maps $T(M)$ to itself and $f$ is a local homeomorphism near $z$.

For the second property, we start with $M \subset V_{T(M)} \subset T(M)=[M]$, and we trivially get $[M] \subset\left[V_{T(M)}\right] \subset T(M)=[M]$ by minimality.

Corollary 2.14. Let $M, M^{\prime} \supset \Omega_{f}$ be finite invariant sets. If $V_{T(M)}=$ $V_{T\left(M^{\prime}\right)}$, then $T(M)=T\left(M^{\prime}\right)$.

The following expanding property is characteristic of dynamical trees coming from postcritically finite polynomials.

Proposition 2.15. Let $v, v^{\prime} \in J(f) \cap T(M)$ be periodic. If for all $n \geq 0$ the points $f^{\circ n}(v)$ and $f^{\circ n}\left(v^{\prime}\right)$ belong to the same member of $\tilde{T}(M)$, then $v=v^{\prime}$. 
Proof. The hypothesis implies two noteworthy facts. First, for all $n$ the regulated $\operatorname{arcs}\left[f^{\circ n}(v), f^{\circ n}\left(v^{\prime}\right)\right]$ contain no critical points in their interiors. Second, in light of Lemma 2.5, all these intervals are pairwise homeomorphic. If we also assume $v \neq v^{\prime}$, then the interval $\left[v, v^{\prime}\right]$ avoids all Fatou components, since otherwise some iterate will eventually reach a Fatou critical point. Next, let $m$ be a high common multiple of the periods of $v$ and $v^{\prime}$. In this way, by uniqueness of regulated paths, $f^{\circ m}$ maps the interval $\left[v, v^{\prime}\right]$ into itself. By restriction we can assume that there are no further periodic points of period $m$ in the interval. But $v$ and $v^{\prime}$ are repelling for $f^{\circ m}$, so it is topologically impossible not to find a third fixed point for $f^{\circ m}$ inside $\left[v, v^{\prime}\right]$.

REMARK 2.16. The same is true if $v, v^{\prime}$ are taken only preperiodic. In fact, high iterates of them are eventually equal, and then Proposition 2.12 (or Lemma 2.5) implies equality for $v, v^{\prime}$.

We define the tree distance $\operatorname{dist}_{T(M)}\left(v, v^{\prime}\right)$ for $v, v^{\prime} \in V_{T(M)}$ as the number of edges between $v$ and $v^{\prime}$ (in $\left[v, v^{\prime}\right]$ ). We rephrase Proposition 2.15 in a more combinatorial way.

TheOREM 2.17 (Expanding property of Hubbard trees). If $v, v^{\prime} \in V_{T(M)}$ $\cap J(f)$ satisfy $\operatorname{dist}_{T(M)}\left(v, v^{\prime}\right)=1$, then $\left.\operatorname{dist}_{T(M)}\left(f^{\circ n}(v)\right), f^{\circ n}\left(v^{\prime}\right)\right)>1$ for some $n \geq 1$.

Proof. If the distance between successive iterates of $v, v^{\prime}$ is 1 , they belong to the same member of $\tilde{T}(M)$. Because of Proposition 2.15 (really, of Remark 2.16) at some point this no longer holds and the distance is greater than 1.

3. Inverses and incidence. In this section we reconstruct the inverse of a Hubbard tree given knowledge of the set of vertices, its preferred set. And in fact, the tree generated by the inverse of an invariant set that includes the critical points matches the inverse tree. This makes it possible to understand how the incidence grows as we enlarge the tree.

LEMMA 3.1. For $M \supset \Omega_{f}$ finite and invariant we have $f^{-1} T(M)=$ $T\left(f^{-1} M\right)=T\left(f^{-1} V_{T(M)}\right)$. The set of vertices of this new tree is given by $V_{T\left(f^{-1} M\right)}=f^{-1} V_{T(M)}$.

Proof. Let us travel around the chain of inclusions. Take $z \in f^{-1} T(M)$. If $z \in f^{-1} M$, then we also have $z \in T\left(f^{-1} M\right)$. Else $z \notin f^{-1} M$, so $f(z)$ is in $T(M)$ but not in $M$. Hence $f(z)$ belongs to a regulated arc that touches $M$ only at the extremes. By Lemma 2.6 any inverse of this regulated arc is also regulated and has endpoints in $f^{-1} M$. Therefore $z$ is in $T\left(f^{-1} M\right)$, and we obtain $f^{-1} T(M) \subset T\left(f^{-1} M\right)$.

Starting from $f^{-1} M \subset f^{-1} V_{T(M)}$ we get $T\left(f^{-1} M\right) \subset T\left(f^{-1} V_{T(M)}\right)$ for free. 
Finally, as the invariant set $f^{-1} V_{T(M)}$ includes all critical points, Lemma 2.11 implies $f T\left(f^{-1} V_{T(M)}\right)=\left[f f^{-1} V_{T(M)}\right]=\left[V_{T(M)}\right]=[M]$.

For the extra property, note that except at critical points (which are already in $M, V_{M}, f^{-1} M$ and $\left.V_{T\left(f^{-1} M\right)}\right)$, everything near $v$ in $f^{-1} T(M)$ replicates around $f(v)$ in $T(M)$ via $f$. In particular, for incidence numbers we have $\nu_{f^{-1} T(M)}(v)=\nu_{T(M)}(f(v))$ at any regular $v$. This is enough to certify that preimages of branching points remain branching in the inverse tree and that no new unexpected branching points appear. In other words, we have established the equality $V_{T\left(f^{-1} M\right)}=f^{-1} V_{T(M)}$.

Corollary 3.2. For $M \supset \Omega_{f}$ finite and invariant we have $f^{-n} T(M)=$ $T\left(f^{-n} M\right)=T\left(f^{-n} V_{T(M)}\right)$. The set of vertices in the corresponding inverse is given by $V_{T\left(f^{-n} M\right)}=f^{-n} V_{T(M)}$.

As we always have $T(M) \subset T\left(f^{-1} M\right)$, there are several incidence functions to be considered: one for each tree. Write $\nu_{n, M}(z)$ for the incidence at $z$ in the tree $f^{-n} T(M)$ whenever this makes sense. Since the incidence can only grow, these values satisfy $\nu_{0, M}(z) \leq \nu_{1, M}(z) \leq \cdots$. Dynamically they also keep some logic.

Lemma 3.3. For every $z \in f^{-1} T(M)$ we have

$$
\nu_{1, M}(z)=\nu_{0, M}(f(z)) \operatorname{deg}_{z} f .
$$

Proof. This is an easy consequence of Lemma 3.1.

The next proposition is a coarse attempt to reconstruct $f^{-1} T(M)$ from $T(M)$. A subtler version will be given later, in Section 8 .

Lemma 3.4. Let $X$ be a member of $\widetilde{T}\left(f^{-1} M\right)$. Then $f$ induces a homeomorphism between $X$ and the component of $T(M)$ cut open along $f\left(X \cap \Omega_{f}\right)$ that contains $f(X)$.

Proof. By Proposition 2.12, $f$ restricted to $X$ is injective. Furthermore, $f(X)$ is relatively open in the said component. As it is also compact, it must be the whole component.

Lemma 3.3 describes how the incidence number behaves dynamically as the tree grows. For the rest of this section we take a careful look at terminal, incidence, branching and non-branching points. The potential discrepancy on the numbers depends on whether we stand in the filled Julia set $K(f)$, in a Hubbard tree $T(M)$ or in one of its inverses $f^{-n} T(M)$.

A point $p \in J(f)$ is terminal if there is only one external ray landing at $p$. Else $p$ is an incidence point. For incidence points we specialize between branching (more than two rays land at $p$ ) and nonbranching (exactly two rays land at $p$ ). As we will soon see, in the postcritically finite case every branching point is periodic or preperiodic. Also, every periodic incidence 
point is present as a preferred member of the minimal tree $T\left(\mathcal{O}\left(\Omega_{f}\right)\right)$, and hence appears in any $T(M)$.

Pick $z \in J(f)$. By general principles, every component of $J(f)-\{z\}$ eventually gets mapped onto the full Julia set, and therefore contains points whose orbit catches up with any prescribed element in $J(f)$ (cf. [M]). This fact will be used in conjunction with the following technical tool.

Lemma 3.5. Take $M \supset \Omega_{f}$ finite and invariant. Suppose $N \supset \Omega_{f}$ is also invariant and satisfies $f^{\circ n}(N) \subset M$. Then $f^{\circ n} T(N) \subset T(M)$.

Proof. Because $N \subset f^{-n}(M)$, we get $T(N) \subset T\left(f^{-n}(M)\right)=f^{-n}(T(M))$ thanks to Corollary 3.2.

Proposition 3.6. Let $f$ be postcritically finite and $z \in J(f)$ a branching point. Then $z$ is periodic or preperiodic.

Proof. We consider only $z$ outside $\mathcal{O}\left(\Omega_{f}\right)$ (else $z$ is already preperiodic). Fix $w \in \Omega_{f}$ and grab from three different components of $J(f)-\{z\}$ points $p_{1}, p_{2}, p_{3}$ that sooner or later map to $w$. As the orbit $\mathcal{O}\left(\left\{p_{1}, p_{2}, p_{3}\right\}\right)$ is finite, the invariant set $N=\mathcal{O}\left(\Omega_{f}\right) \cup \mathcal{O}\left(\left\{p_{1}, p_{2}, p_{3}\right\}\right)$ qualifies to generate a Hubbard tree. In $T(N)$ the incidence at $z$ is at least three, which makes it a preferred vertex. But $V_{T(N)}$ is finite and invariant, so we are done.

Proposition 3.7. Let $z \in J(f)$ be a periodic incidence point. Then $z$ appears in the minimal tree $T\left(\mathcal{O}\left(\Omega_{f}\right)\right.$ ) (and hence in all Hubbard trees). Furthermore, the incidence number $\nu_{T(M)}(z)$ is independent of $M$ and coincides with the number of components of $J(f)-\{z\}$. In particular, exactly $\nu_{T(M)}(z)$ rays land at $z$.

Proof. Once we remember that the number of rays landing at $z$ coincides with the number of connected components of $J(f)-\{z\}$, the rest of the proof reproduces the scheme of the last proposition. Let us be precise. In each component of $J(f)-\{z\}$ we choose an element that eventually maps to a critical point. Next we form the Hubbard tree associated with the union of the marked set and the orbits of the appended elements. Lemma 3.5 explains why this augmented tree is swallowed by the original one under iteration. The result now follows from the fact that, by Lemma 3.3, incidence numbers at periodic Julia vertices do not decrease after iteration. Further details are left to the reader.

Corollary 3.8. Let $z \in J(f) \cap T(M)$ be such that $f^{\circ n}(z)$ is periodic. Then $\nu_{n, M}(z)$ equals the number of components of $J(f)-\{z\}$. In particular, exactly $\nu_{n, M}(z)$ external rays land at $z$.

Proof. This follows from Proposition 3.7 and Lemma 3.3.

Corollary 3.9. Every tree $T(M)$ contains a fixed point of $f$. 
Proof. Let $d$ be the degree of $f$. If $f$ admits a fixed critical point, then this point belongs to $T(M)$. Else, as there are only $d-1$ fixed rays but $d$ fixed points, one of them must have incidence. By Proposition 3.7 it already appears in $T(M)$.

4. Examples and counterexamples. In this section we present examples of actual Hubbard trees. In particular we settle the case of the tree consisting of a unique critical vertex. We also justify the importance of all the items present in the definition. This is done by means of counterexamples where either existence or uniqueness fails. In other words, we explain why further structure needs to be imposed. At the end we make two brief observations indirectly related to uniqueness: we talk about extensions and internal symmetries.

In the diagrams we will illustrate Julia points with filled dots and Fatou points with small circles. Also, critical points will be indicated with as many asterisks as necessary.

EXAMPLE 4.1 (Hubbard trees of dimension 0). When a Hubbard tree has just one vertex, this point maps to itself with degree $d$. For sure, the only centered monic polynomial that realizes this Hubbard tree is $f(z)=z^{d}$. The filled Julia set is the closed unit disk.

From now on, the dynamics in the tree is the edge to edge extension of the vertex dynamics. This is possible under the nondegeneracy hypothesis.

EXAmple 4.2. For the degree two polynomial $f(z)=z^{2}+c$, with $c$ approximately equal to $-0.12+0.74 i$, known as the rabbit, the critical point moves around a fixed point in a period three orbit. As captured by the minimal tree, this is the only interesting dynamical feature.

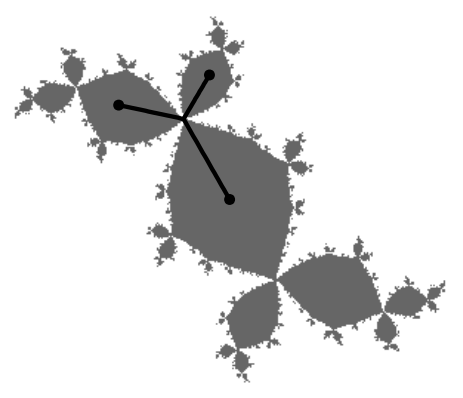

Fig. 4.1. The rabbit with minimal tree inside

EXAmple 4.3. For the dynamical system $z \mapsto z^{2}+i$ (the parameter $c=i$ corresponds to one of the tips of the Mandelbrot set), the critical orbit 
moves as

$$
0 \mapsto i \mapsto-1+i \mapsto-i \mapsto-1+i \mapsto \cdots .
$$

In order to make things a bit more interesting, we take into account also the period three (Julia) orbit $-1.29+0.779 i \mapsto 1.058-1.011 i \mapsto 0.097-1.140 i \mapsto$ $-1.29+0.779 i$ (where the rays of argument $3 / 7,6 / 7,5 / 7$ land). The Hubbard tree generated by all those points is exhibited in Figure 4.2. Precise angles are not mentioned as there are no Fatou critical points.

As a combined consequence of Lemma 3.3 and Proposition 3.6, the highest incidence for Julia set points already occurs in the minimal tree. Therefore, in this particular example all other periodic points either are hidden inside the minimal tree or are ends in some extension. In the latter case the rays and the landing point share period. Now, there are only two period three strings under doubling, namely $1 / 7 \mapsto 2 / 7 \mapsto 4 / 7$ and $3 / 7 \mapsto 6 / 7 \mapsto 5 / 7$. The rays with argument in the first group come together at $z_{4}=-0.3+0.625 i$, a rotation three fixed point. The other group corresponds to the period three orbit just mentioned before. But $f$ has another period three orbit, namely, $0.10-0.349 i \mapsto-0.112+0.930 i \mapsto$ $-0.852+0.792 i \mapsto 0.10-0.349 i$. However, this one cannot be associated with a period 3 ray sequence (simply because the two period three ray orbits are already accounted for) and as such is not composed of ends. Hence, this orbit lives inside the minimal tree. Where? Let us see. The tree is expanding, so it should be a simple matter for those familiar with Markov partition techniques to place correctly the orbit inside our graph. This is an easy exercise for our readers.
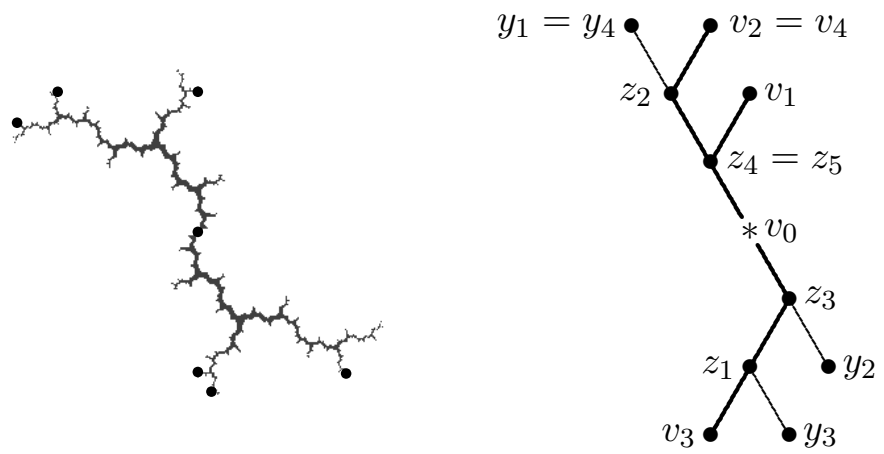

Fig. 4.2. The minimal tree - thick - is recovered by restricting to the regulated hull of the critical orbit and then erasing unessential vertices. If we rescale some branches and slightly rotate near nodes, the resemblance to the actual tree is evident.

EXAMPLE 4.4. Take $f(z)=0.25 z^{4}-(1.24312-0.434889 i) z^{3}+(0.345782$ $-0.255665 i) z^{2}$. This postcritically finite polynomial has two periodic crit- 
ical points with orbits $0 \mapsto 0$ and $-1.24565+0.339367 i \mapsto 0.261681-$ $0.677055 i \mapsto-1.24565+0.339367 i$, and one preperiodic Julia critical point with orbit $-2.48372+0.965301 i \mapsto 0.410426+0.189372 i \mapsto 0.410426+$ $0.189372 i$. The Julia set is given in Figure 4.3. The abstract Hubbard tree is exhibited in Figure 6.1. Its inverse tree is shown in Figure 8.5.

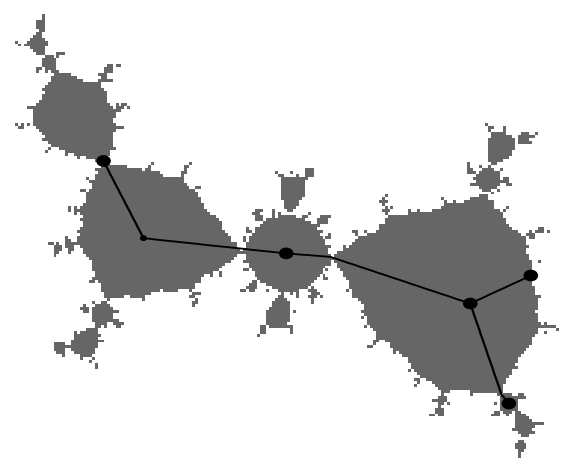

Fig. 4.3

Now we analyze why further conditions are needed in order to guarantee a complete characterization.

EXAMPLE 4.5 (Nonuniqueness). Let us take the following minimal tree and try to realize it by a degree three polynomial. (The double star stands for a double critical point, that is, of local degree 3.)

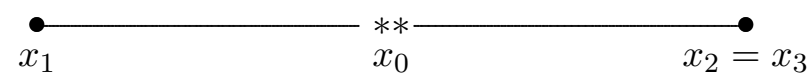

Fig. 4.4. The dynamics is given by $x_{0} \mapsto x_{1} \mapsto x_{2} \mapsto x_{3}=x_{2}$.

All degree three polynomials with a double critical point are conjugated to $z \mapsto f_{c}(z)=z^{3}+c$ for some $c$, that is, we can assume directly $x_{0}=0$. Remember also that for polynomials of shape $f_{c}(z)=z^{3}+c$, the value $c^{2}$ represents a complete invariant: $f_{c}$ and $f_{c^{\prime}}$ are conjugated if and only if $c^{2}=c^{\prime 2}$.

As the critical dynamics is given by $0 \mapsto c \mapsto c^{3}+c \mapsto c^{3}+c$, the relation $f_{c}^{\circ 2}(0)=f_{c}^{\circ 3}(0)$ is equivalent to $c^{3}+c=\left(c^{3}+c\right)^{3}+c$. Thus, the parameter $c$ satisfies $c^{5}\left(c^{4}+3 c^{2}+3\right)=0$. But $c^{3}+c \neq 0$ (unless we assume $x_{2}=x_{0}$ ) implies $c \neq 0$, and we grab two different acceptable values for $c^{2}$. For these two values of $c^{2}$ the minimal tree is pictured in Figure 4.4. In fact, 
Proposition 2.15 implies that $f(0)$ and $f^{\circ 2}(0)$ belong to different components of $T-\{0\}$. Nevertheless, the "inverse trees" — determined by $f^{-1}\left(\Omega_{f}\right)$ - are quite different. They are sketched below.
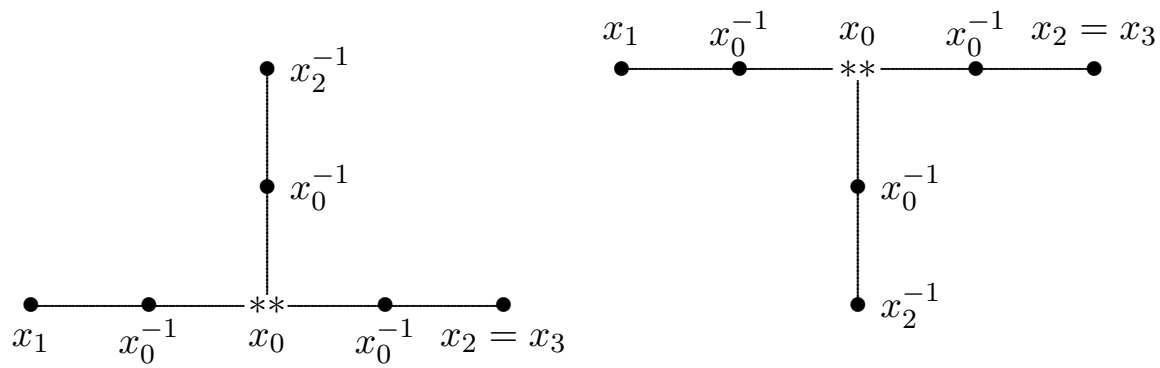

Fig. 4.5. The newly added points $v^{-1}$ map to $v$. Even if the two trees are isomorphic, they fail to have the same cyclic order around $x_{0}$. Since there are no Fatou points, the disposition of the edges is enough and there is no need for precise angles.
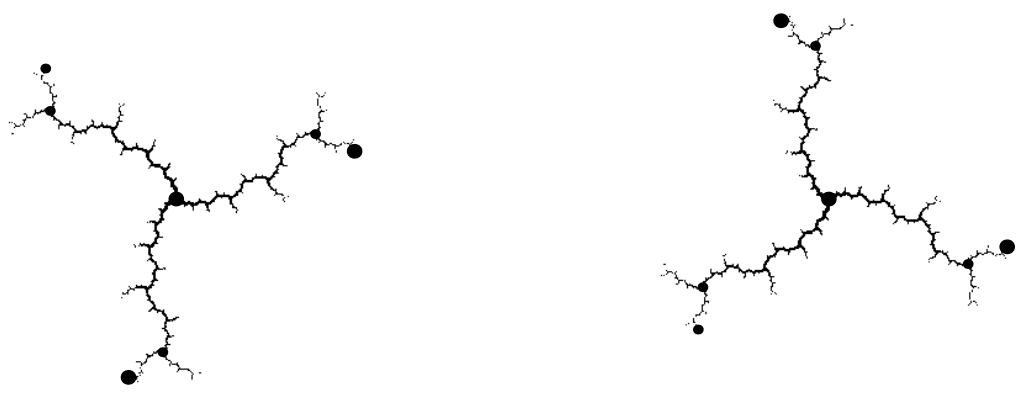

Fig. 4.6. The actual Julia sets

Now we draw attention to two typical cases where existence fails.

ExAmple 4.6 (Nonexistence). The tree below cannot be obtained from a polynomial map. In fact, there is no degree two polynomial with three fixed points.

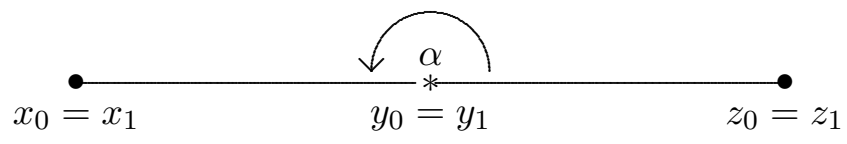

Fig. 4.7. All vertices are fixed. Local degrees from left to right are 1, 2, 1.

There is a further obstruction to realizing this tree. If this tree were to come from a degree two polynomial $f$, then the edges incident at the 
fixed critical point should materialize as internal rays in the uniformizing coordinate. Let $\alpha$ be the angle between the segments. After applying $f$ we obtain $2 \alpha \equiv \alpha(\bmod 1)$, an equation that can only be solved with $\alpha \equiv 0$ $(\bmod 1)$, and the two segments must be identified.

EXAMPLE 4.7 (Nonexistence). The following minimal tree cannot be obtained from a polynomial map as it fails to satisfy the expanding condition.

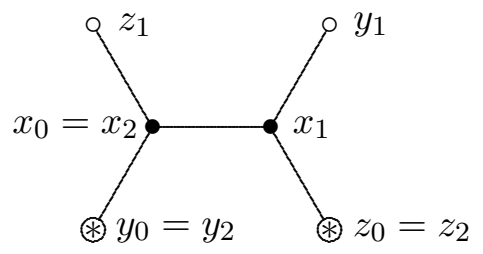

Fig. 4.8. For any $k$ there is no vertex between $f^{\circ k}\left(x_{0}\right)$ and $f^{\circ k}\left(x_{1}\right)$.

All that can go wrong already happened in the last three examples. In Example 4.5, uniqueness failed due to lack of information to recover the tree $f^{-1} T(M)$. In the other two examples we were unable to comply with necessary conditions: the trees must carry precise angles around Fatou critical vertices and adjacent Julia vertices should eventually spread apart.

EXAMPLE 4.8 (Compare Example 4.2). Now we want to consider a couple of extensions of the minimal tree of the rabbit (Figure 4.9, left).

In Figure 4.9, middle, we are extending the tree so that now it includes a second fixed point, the place where the ray of argument zero lands.
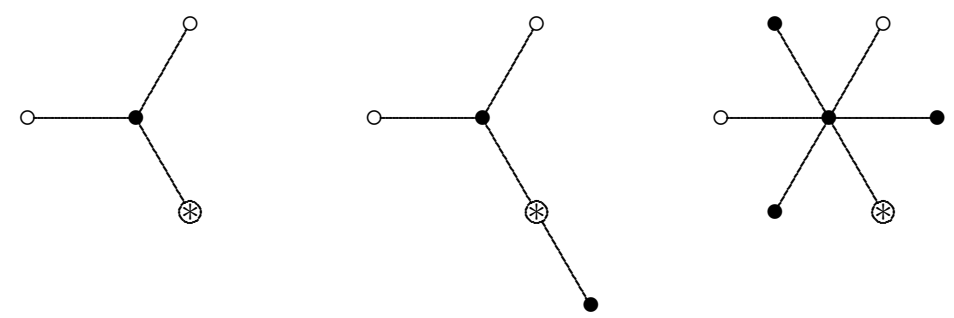

Fig. 4.9. The minimal rabbit and two extensions. One works and the other does not.

On the right, however, we have an unfeasible extension. Even if the old angles are preserved, the presence of nonexpanding pairs spoils everything. In fact, there are multiple reasons to disregard this dynamics as a Hubbard tree. For example, the newly appended orbit lives too close to the fixed points. Also, around the Julia fixed point we detect branches disjoint from the critical orbit (compare Theorem 5.9). Of course, the latter violation implies failure to comply with the expanding condition. 
As a consequence of uniqueness, an extension of a dynamical tree has even less chance to be realized than the original one. In practice, we must be careful with what we want to call a "legal extension". Extensions must be canonical in the sense that they can be reconstructed from the minimal data.

EXAMPLE 4.9. If we flip a Hubbard tree $H$ about the $x$-axis, we get a new dynamical tree $\bar{H}$. Each element has a mirror image. For example a vertex $v \in H$ corresponds to $\bar{v} \in \bar{H}$, an edge $e$, to $\bar{e}$. The vertex dynamics is given by $\bar{f}(\bar{v})=\overline{f(v)}$. Degrees do not change. The angle satisfies $\angle\left(\bar{e}, \bar{e}^{\prime}\right)=-\angle\left(e, e^{\prime}\right)$, etc. Clearly, the expanding condition still holds, so we have a sound Hubbard tree. If $H$ is realized by $F$ then $\bar{H}$ is realized by the conjugate $\bar{F}$ defined as $\bar{F}(z)=\overline{F(\bar{z})}$.

This is important because everything that we can say or build using a given orientation remains valid for the opposite. A simple application is the following. If $H$ itself is symmetric by reflection about the $x$-axis, then $H$ can be realized by a real polynomial. In fact, in this case $H$ and $\bar{H}$ are the same, so uniqueness implies $F=\bar{F}$.

5. Abstract Hubbard trees. Now we study abstract Hubbard trees as defined in the introduction. The Hubbard tree with a single vertex was solved as $z \mapsto z^{d}$ in Example 4.1. Henceforth we assume that our trees are one-dimensional. Two recurrent features in the abstract theory are our capability to assign "Böttcher" coordinates around Fatou points and the presence of an expanding condition for adjacent Julia elements.

Whenever $e$ is an edge incident at $v$, inside the set $f(e)$ we can find a unique edge with $f(v)$ as an endpoint. By abuse of notation we still call this edge $f(e)$. As we are talking about the induced action on the edges around a vertex, this should cause no confusion.

We first simplify the local model around periodic Fatou points. When $v_{0} \mapsto v_{1} \mapsto \cdots \mapsto v_{m}=v_{0}$ is a period $m$ critical cycle, its global degree is the product $d\left(v_{0}\right) \cdots d\left(v_{m-1}\right)$ of individual degrees.

A Böttcher coordinate along a critical cycle is an assignment $\varphi_{v}(e) \in$ $\mathbb{T}=\mathbb{R} / \mathbb{Z}$ (for all edges incident at vertices in the loop) so that $\angle_{v}\left(e, e^{\prime}\right)=$ $\varphi_{v}\left(e^{\prime}\right)-\varphi(e)$ and $\varphi_{f(v)}(f(e))=d(v) \varphi_{v}(e)$.

Lemma 5.1. For a critical cycle of global degree $k$ there are precisely $k-1$ ways to assign Böttcher coordinates.

Proof. If $m$ is the length of the cycle, then $f^{\circ m}$ sends germs of edges at $v$ to germs of edges at $v$. Hence, the presence of a good Böttcher coordinate for $e$ is tantamount to the equality $\varphi_{v}\left(f^{m}(e)\right)=k \varphi_{v}(e)$. By definition we then have

$$
\angle_{v}\left(f^{\circ m}(e), e\right) \equiv(k-1) \varphi_{v}(e)(\bmod 1) .
$$


There are exactly $k-1$ ways to solve this equation for $\varphi_{v}(e)$ since the value $\angle_{v}\left(f^{\circ m}(e), e\right)$ is known a priori.

Once a value $\varphi_{v}(e)$ is known, all other assignments are found via the formula $\varphi_{v}\left(e^{\prime}\right)=\varphi(e)+\angle_{v}\left(e, e^{\prime}\right)$. Following the same trend, if we define $\varphi_{f(v)}(f(e))=d(v) \varphi_{v}(e)$, everything is completed in a compatible way along the cycle.

We can inductively assign coordinates at nonperiodic Fatou vertices in the usual style. Fix an edge $e$ incident at $v$ and set $\varphi_{v}(e)=\varphi(f(e)) / d(v)$. For other vertices write $\varphi\left(e^{\prime}\right)=\varphi(e)+\angle_{v}\left(e^{\prime}, e\right)$. This is again a Böttcher coordinate.

Our abstract definition already absorbs the essence of "honest" Hubbard trees. This makes the basic properties of abstract trees even easier to handle.

Remember that thanks to the nondegeneracy condition, the vertex dynamics $f: V \rightarrow V$ extends naturally to the whole tree.

Lemma 5.2. Suppose the segment $\left[z_{1}, z_{2}\right] \subset H$ contains no critical point of $f$, except, perhaps, for the endpoints. Then $f$ is injective when restricted to $\left[z_{1}, z_{2}\right]$, and we have $f\left(\left[z_{1}, z_{2}\right]\right)=\left[f\left(z_{1}\right), f\left(z_{2}\right)\right]$.

Proof. (A no-frills version of Lemma 2.5.) Let $\gamma: I \rightarrow H$ be the injective map that defines the arc $\left[z_{1}, z_{2}\right]$. We will focus on proving that $f \circ \gamma$ is one-to-one. As $f \circ \gamma$ is locally injective, the set $\Delta=\left\{\left(t_{1}, t_{2}\right)\right.$ : $t_{1}<t_{2}$ and $\left.f\left(\gamma\left(t_{1}\right)\right)=f\left(\gamma\left(t_{2}\right)\right)\right\}$ is compact. In case $\Delta$ were nonempty, there would be $\left(t_{1}, t_{2}\right) \in \Delta$ with $t_{2}-t_{1}>0$ minimal. In particular, for every $s<t$ with $t-s<t_{2}-t_{1}$ the composition $f \circ \gamma$ determines an arc with domain $[s, t]$. Whenever we have $t \in\left(t_{1}, t_{2}\right)$, the restriction of $f \circ \gamma$ to both $\left[t_{1}, t\right]$ and $\left[t, t_{2}\right]$ gives two different arcs between $f(\gamma(t))$ and $f\left(\gamma\left(t_{1}\right)\right)=f\left(\gamma\left(t_{2}\right)\right)$. This is impossible in a tree.

Write $\Omega_{H}$ for the critical set of the dynamical tree. For a subset $W \subset V$ of vertices, let $[W]_{H}$ (or $[W]$ ) be the smallest subtree of $H$ that contains $W$. Of course, by definition we already have $H=[V]_{H}$. The idea now is to state-no proofs are necessary - analogous properties to those given in Section 2 for Hubbard trees.

Proposition 5.3. For any subset $W \subset V$ we have $[f(W)] \subset f([W]) \subset$ $\left[f\left(W \cup \Omega_{H}\right)\right]$.

Lemma 5.4. If an invariant set $M \subset V$ contains $\Omega_{H}$, then $f([M])=$ $[f(M)] \subset[M]$.

In order to understand the dynamics in a Hubbard tree, we restrict to portions where $f$ is injective. As in Section 2, we write $\widetilde{H}$ for the family whose members are the closures of the components of $H-\Omega_{H}$. 
Proposition 5.5. The dynamics $f$ induces a continuous map on $H$ whose restriction to every piece of $\widetilde{H}$ is injective.

There is no analog to Proposition 2.15 as it is technically equivalent to Theorem 2.17, the expanding condition. Recall from the introduction that the distance $\operatorname{dist}_{H}\left(v, v^{\prime}\right)$ between vertices in $H$ counts the number of edges in $\left[v, v^{\prime}\right]$. Our tree is expanding if for every edge $e$ whose endpoints $v, v^{\prime}$ are Julia vertices there is $n \geq 1$ such that $\operatorname{dist}_{H}\left(f^{\circ n}(v), f^{\circ n}\left(v^{\prime}\right)\right)>1$. Evidently, it is enough to check this condition for adjacent periodic Julia vertices.

We prove that a contextual equivalence of Proposition 2.15 and Theorem 2.17 is also valid.

Proposition 5.6. An angled tree is expanding if and only if given two Julia vertices $v, v^{\prime}$ there is $n \geq 0$ such that $f^{\circ n}(v)$ and $f^{\circ n}\left(v^{\prime}\right)$ do not belong to the same piece of $\widetilde{H}$. Even more, it is enough to test this property for consecutive periodic vertices to decide whether or not the tree is expanding.

Proof. Suppose the tree is not expanding. Then there are contiguous periodic Julia vertices $v, v^{\prime}$ with $\operatorname{dist}_{H}\left(f^{\circ n}(v), f^{\circ n}\left(v^{\prime}\right)\right)=1$ for all $n \geq 0$. Clearly there is a member of $\widetilde{H}$ where we can find those pairs together.

Conversely, take an expanding tree. By contradiction suppose there are different Julia vertices $v, v^{\prime}$ whose successive iterates remain tied to a common member of $\widetilde{H}$. Among such pairs pick one with maximal $\operatorname{dist}_{H}\left(v, v^{\prime}\right)$. By assumption, for all $n \geq 0$, the arc $\left[f^{\circ n}(v), f^{\circ n}\left(v^{\prime}\right)\right]$ is contained within a component of $\widetilde{H}$. It follows from Lemma 5.2 and maximality that all such intervals are naturally homeomorphic to each other. We take $v^{\prime \prime} \in\left[v, v^{\prime}\right] \cap V$ such that $\operatorname{dist}\left(v, v^{\prime \prime}\right)=1$. Since $f$ maps vertices to vertices, we must have $\operatorname{dist}_{H}\left(f^{n}(v), f^{n}\left(v^{\prime \prime}\right)\right)=1$, for all $n$. As the tree is expanding and $v$ is of Julia type, necessarily $v^{\prime \prime}$ is a Fatou vertex. In particular, $v^{\prime \prime}$ is different from $v^{\prime}$. From this we get a contradiction as $v^{\prime \prime}$, being a Fatou vertex, is due sooner or later to hit a critical point $f^{k}\left(v^{\prime \prime}\right)$ that will block $f^{k}(v)$ from $f^{k}\left(v^{\prime}\right)$.

REMARK 5.7. This last proposition should be regarded as a distance-free version of the expanding condition. It will prove helpful when we enlarge or restrict the tree.

Whenever $e$ is an edge incident at $v$, let $\mathcal{B}_{v}(e)$ be the branch that stems from $e$, that is, the closure of the connected component of $H-\{v\}$ that contains $e$. Denote by $\mathcal{N}_{v}(e)$ the number of vertices found in $\mathcal{B}_{v}(e)$ (including $v$, by convention). Recall that in this context $f(e)$ refers to the only edge one of whose endpoints is $f(v)$ and which as a set is contained in $f(e)$.

LEMma 5.8. If no critical point belongs to $\mathcal{B}_{v}(e)$, then $f$ is injective in this branch and satisfies $f\left(\mathcal{B}_{v}(e)\right) \subset \mathcal{B}_{f(v)}(f(e))$. In particular, we have $\mathcal{N}_{v}(e) \leq \mathcal{N}_{f(v)}(f(e))$. 
Proof. If $\left[z_{0}, z_{1}\right]$ lies in $\mathcal{B}_{v}(e)$, then Lemma 5.2 implies $f\left(z_{0}\right) \neq f\left(z_{1}\right)$ (unless $z_{0}=z_{1}$, an uninteresting case). Taking $z_{0}=v$ we get $e \subset\left[v, z_{1}\right]$, and therefore also $f(e) \subset\left[f(v), f\left(z_{1}\right)\right]$. This fact together with the injectivity of $f$ in $\left[v, z_{1}\right]$ is enough to recognize that $\left[f(v), f\left(z_{1}\right)\right]$ is trapped inside $\mathcal{B}_{f(v)}(f(e))$.

The extra property is true because vertices map to vertices.

The next result represents more than the abstract counterpart to Propositions 3.6 and 3.7. It also tells us that the incidence at periodic Julia points should be kept unaltered for the expanding condition to hold in an extension.

THeOREM 5.9. If $v$ is a periodic Julia vertex, then every branch at $v$ intersects $\mathcal{O}\left(\Omega_{H}\right)$.

Proof. As $v$ is a regular point (for otherwise it is of Fatou type), we can suppose that $\mathcal{B}_{v}(e)$ contains no critical point (else there is nothing to prove).

Since along the orbit of $v$ matters are one-to-one, there is a periodic sequence of pairs $\left(v_{0}, e_{0}\right), \ldots,\left(v_{m-1}, e_{m-1}\right)$, where $e_{i}$ is an edge incident at $v_{i}$, subject to $f\left(v_{i}\right)=v_{i+1}$ and $f\left(e_{i}\right)=e_{i+1}$. (Here $v_{i}=v_{j}$ does not imply $e_{i}=e_{j}$ : take for example a fixed point with nontrivial rotation number.)

If for some $l<m$ the branch $\mathcal{B}_{f^{\circ l}(v)}\left(e_{l}\right)$ shelters a critical point, we take $l$ as large as possible, that is, we also assume that no critical point appears in $\mathcal{B}_{f^{\circ l+1}(v)}\left(e_{l+1}\right), \ldots, \mathcal{B}_{f^{\circ m-1}(v)}\left(e_{m-1}\right)$. Pick $\omega \in \mathcal{B}_{f^{\circ l}(v)}\left(e_{k}\right)$ critical. By choosing perhaps some other critical point in the same branch we can assume the absence of further critical points in $\left[f^{\circ l}(v), \omega\right]$. We first get directly $\left[f^{\circ l+1}(v), f(\omega)\right] \subset \mathcal{B}_{f^{\circ l+1}(v)}\left(e_{l+1}\right)$ since $f$ is injective in $\left[f^{\circ l}(v), \omega\right]$, and then inductively $\left[f^{\circ l+i}(v), f^{i}(\omega)\right] \subset \mathcal{B}_{f^{\circ l+i}(v)}\left(e_{l+i}\right)$ for $i=2, \ldots, m-l$, after applying Lemma 5.8. In particular, $f^{\circ m-l}(\omega) \in \mathcal{B}_{f^{\circ m}(v)}\left(e_{m}\right)=\mathcal{B}_{v}(e)$, and we are done.

Otherwise, if $\mathcal{B}_{f^{\circ l}(v)}\left(e_{l}\right)$ contains no critical point for all $l$, the branches are homeomorphic in view of Lemma 5.8 again. Therefore within each branch every vertex is periodic. As they are non critical, they are of Julia type. Here we get plenty of nonexpanding pairs and a contradiction.

Extensions of trees might be hard to construct but they are nonetheless easy to define. We have to enlarge the tree and in the new graph manipulate a bigger set of vertices. As we do not want to allow extensions that increase the global degree, at each new vertex $v$ we must set $d(v)=1$. Preexisting angles (between old edges) are supposed to be preserved. When all these together with the expanding condition between Julia vertices (of the extended dynamics) are satisfied we have an extension of $H$.

Reciprocally, we say that one tree is the restriction of another if the latter is an extension of the former. 
When thinking of extensions and restrictions, special attention must be paid to Julia vertices: by definition there is a tight relation between the value of the angles measured at $v$ and the number of components that make the complement of $v$. (Compare Theorem 5.9 and Example 4.8.)

Regarding the expanding condition, when just new Fatou or preperiodic Julia vertices are appended to an expanding tree, then the extension is trivially expanding. This happens because no new consecutive periodic Julia pairs ought to be tested for an obstruction.

Theorem 2.17 reduces our interest to expanding trees. For that reason, only expanding extensions will be studied. Among them, we focus on those we call canonical. The idea is that the original tree and the extension convey the same essential data. To be precise, an extension of $H$ is canonical if given another [expanding] extension of $H$ there exists a common expanding extension of both (and therefore of all three).

For expanding trees we have three trivial dynamical results.

Proposition 5.10. A restriction of an expanding abstract tree is expanding.

Proof. This is a corollary to Proposition 5.6.

Proposition 5.11. Let $H$ be an expanding abstract tree. The number of edges incident at a periodic incidence Julia vertex is the same in $H$ and in any restriction of $H$.

Proof. Because of the last proposition, any restriction of $H$ is also expanding. According to Theorem 5.9 in both cases we are talking about the number of branches that intersect $\mathcal{O}\left(\Omega_{H}\right)$.

An expanding Hubbard tree is minimal if it is generated by the orbit of the critical set. Therefore, every Hubbard tree is an extension of a unique minimal tree.

REMARK 5.12. A postcritically finite polynomial $f$ and a finite invariant set $M \subset \Omega_{f}$ define an abstract Hubbard tree $H_{f, M}$. We recall how the angle function is defined. At Fatou periodic vertices the edges of the tree are radial segments in a Böttcher coordinate: we measure the angle using this chart. Near a nonperiodic Fatou vertex we pull back the ones previously defined. For a Julia set point $v$, the set $J(f)-\{v\}$ consists of a finite number of components, say $m$. The "angle" between two components is the adequate multiple of $1 / \mathrm{m}$.

6. Chopping the trees. Previously we attempted to subdivide the trees by cutting along the critical set. However, the correct way to cut the tree is less radical (but by no means unique). 
In this section we construct a subdivision of $H$, denoted by $T^{*}$, consisting of $d=\operatorname{deg}(H)$ subtrees of $H$ (counting possible repetitions). This partition will have the property that every $p$ in $H$ belongs to $d(p)$ pieces. To achieve this, the tree is to be somehow unglued near the critical points. We first label the critical set as $\Omega_{H}=\left\{v_{1}, \ldots, v_{l}\right\}$.

Start by writing $H_{0}=\{H\}$. We inductively define partitions $H_{i}(i \leq l)$ of $H$ so that for $j \leq i$ the critical vertex $v_{j}$ belongs to exactly $d\left(v_{j}\right)$ constituents of $H_{i}$, and for $j>i$ the vertex $v_{j}$ belongs to just one member of $H_{i}$. The partition $H_{i}$ is formed from $H_{i-1}$ by replacing the only member $T \in H_{i-1}$ to which $v_{i}$ belongs with $d\left(v_{i}\right)$ subtrees of $T$ as indicated below.

Fix an edge $e_{i}$ incident at $v_{i}$. For $k=1, \ldots, d\left(v_{i}\right)$, write $E_{k}=\left\{e^{\prime}\right.$ : $\left.\angle_{v_{i}}\left(e^{\prime}, e_{i}\right) \in[(k-1) / d, k / d)\right\}$. Notice that from where $e_{i}$ stands, we are grouping edges clockwise. This seemingly unnatural choice will pay later on (compare Lemma 11.7). Set

$$
T^{k}=T \cap \bigcup_{e^{\prime} \in E_{k}} \mathcal{B}_{v_{i}}\left(e^{\prime}\right) \cup\left\{v_{i}\right\} .
$$

(To add $v_{i}$ is redundant unless the branch is void.) The new partition $H_{i}$ is defined by removing $T$ from $H_{i-1}$ and including all $T^{k}$. By definition $T^{*}$ is $H_{l}$, the last partition. Clearly, the resulting subtrees depend on the edges $e_{i}$ (here is where uniqueness breaks down) but not on how we sort the critical points.

Example 6.1. Consider the expanding Hubbard tree exhibited in Figure 6.1. The points $A, C, D$ have degree 2 while all others are regular. The relevant dynamical strings are given by $A \mapsto F \mapsto F$, of Julia type, and $C \mapsto E \mapsto C$ and $B \mapsto D \mapsto D$, of Fatou type. The angles are faithfully depicted.

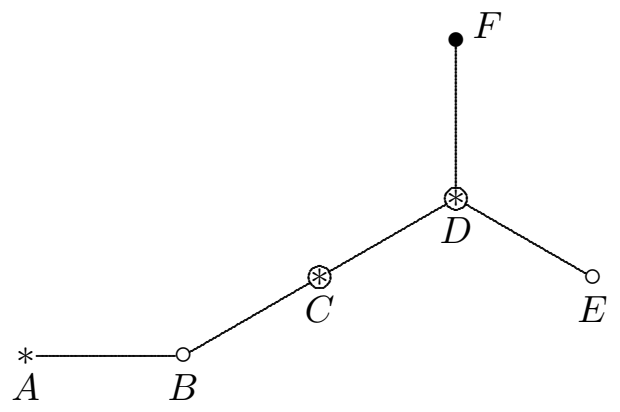

Fig. 6.1

Even if it might seem inefficient, we will follow literally the steps given in the definition. We order the critical elements alphabetically. 
Step 1. Ungluing at $A$. Take $A B$ as reference near $A$ (no other choice). We divide the neighborhood of $A$ into two "balanced" parts: in one we collect the branches that stem clockwise at angles from 0 up to 180 degrees, while in the other those from 180 degrees up to the starting edge (but not including it). The first subtree takes everything. The second must be thought of as a decriticalized copy of $A$.

Step 2. Ungluing at $C$. As $C$ has degree 2, the subtree in which it rests should be cut in two. Choose, say, $C B$ as reference. We collect everything up to the 180 degrees mark, so we only pick the $C B$ branch. The second tree gets the branch growing in the $C D$ direction (and anything else). Up to this point we are left with three trees as shown below.

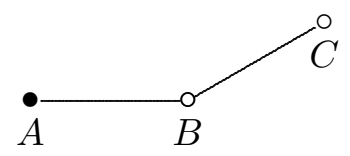

Fig. 6.2

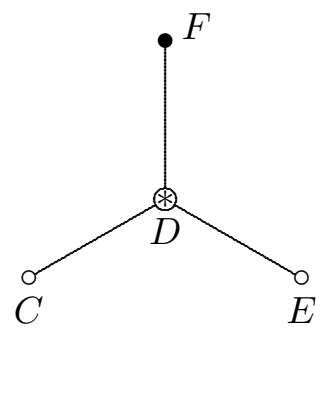

Step 3. Ungluing at $D$. This is the first time we pay attention to $D$, which still lives in a single subtree. According to the definition, the leftmost tree should be split in two. Take the edge $D C$ as reference and start measuring. The first group of branches includes everything before the $180^{\circ}$ mark. This time we obtain a bouquet consisting of the branches pointing toward $C$ and $F$ pinned at $D$. The remainder constitutes the second tree.

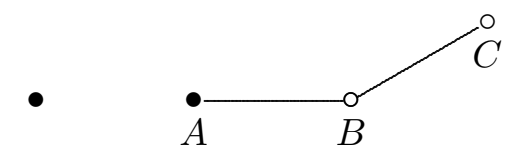

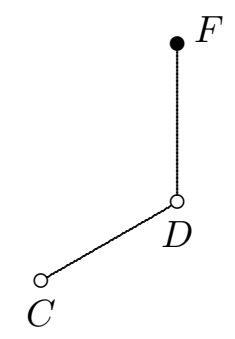

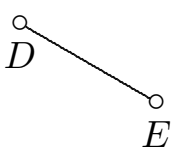

Fig. 6.3 
Lemma 6.2. The vertex dynamics induces a continuous map from the tree to itself where the restriction to every piece of $T^{*}$ is injective.

Proof. Let $T$ be a piece of $T^{*}$. Take an arc $\gamma: I \rightarrow T$ joining different $p_{1}, p_{2} \in T$. We first explain why $\left.f\right|_{T}$ is locally one-to-one also at a potential critical point $c \in T$. At such point all branches stem at arguments inside a wedge not broader than $1 / d(c)$ turns. Therefore no folding takes place around $c$ either. Thus $\Delta=\left\{\left(t_{1}, t_{2}\right): t_{1}<t_{2}\right.$ and $\left.f\left(\gamma\left(t_{1}\right)\right)=f\left(\gamma\left(t_{2}\right)\right)\right\}$ is compact (compare Lemmas 2.5 and 5.2). If we assume $f(\gamma(0))=f\left(p_{1}\right)=$ $f\left(p_{2}\right)=f(\gamma(1))$, then $\Delta$ is nonempty and there exists $\left(t_{1}, t_{2}\right) \in \Delta$ with $t_{2}-t_{1}$ minimal. Let $t \in\left(t_{1}, t_{2}\right)$. Then $f\left(\gamma\left(\left[t_{1}, t\right]\right)\right)$ and $f\left(\gamma\left(\left[t, t_{2}\right]\right)\right)$ are arcs in a tree with the same endpoints. Hence, they are equal, and $t_{2}-t_{1}$ cannot be minimal.

Corollary 6.3. In a degree $d$ Hubbard tree any point has at most $d$ inverses counting multiplicity.

Since each part of $T^{*}$ is a natural place of injectivity, our next task is to improve on Proposition 2.15.

Lemma 6.4. Let e, $e^{\prime}$ be different edges incident at a periodic critical Fatou vertex. Then there is $n \geq 0$ for which $f^{\circ n}(e)$ and $f^{\circ n}\left(e^{\prime}\right)$ belong to different pieces of $T^{*}$.

Proof. Let $k>1$ be the degree of the critical loop $v_{0}=v \mapsto v_{1} \mapsto \cdots \mapsto$ $v_{m}=v_{0}$. Because $k^{l} \angle_{v_{0}}\left(e, e^{\prime}\right)$ blows up to $\infty$ with $l$, there is a first integer $n \geq 1$ for which $d\left(v_{0}\right) d\left(v_{1}\right) \cdots d\left(v_{n}\right) \angle_{v_{0}}\left(e, e^{\prime}\right) \geq 1$. This implies

$$
1 / d\left(v_{n}\right) \leq d\left(v_{0}\right) d\left(v_{1}\right) \cdots d\left(v_{n-1}\right) \angle_{v_{0}}\left(e, e^{\prime}\right)=\angle_{v_{n}}\left(f^{\circ n}(e), f^{\circ n}\left(e^{\prime}\right)\right)<1,
$$

so by definition $f^{\circ n}(e)$ and $f^{\circ n}\left(e^{\prime}\right)$ belong to different pieces of $T^{*}$ determined by the critical point $v_{n}$.

Proposition 6.5. Let $v, v^{\prime}$ be different Julia vertices. For some $n$ the vertices $f^{\circ n}(v)$ and $f^{\circ n}\left(v^{\prime}\right)$ are not in the same element of $T^{*}$.

Proof. We first settle the case where $v, v^{\prime}$ are periodic. Suppose $f$ is not one-to-one near $\omega$ when restricted to the arc $\left[v, v^{\prime}\right]$. Let $e, e^{\prime}$ be the edges incident at $\omega$ that point toward $v, v^{\prime}$, respectively. As around $\omega$ we have folding, the angle $\angle_{\omega}\left(e, e^{\prime}\right)$ is a nontrivial multiple of $1 / d(\omega)$. By construction, the branches determined by $e, e^{\prime}$ always belong to different subtrees in $T^{*}$.

If eventually there is folding between $f^{\circ n}(v)$ and $f^{\circ n}\left(v^{\prime}\right)$, then everything is reduced to the previous case.

Otherwise, if $f$ restricted to the segment $\left[f^{\circ n}(v), f^{\circ n}\left(v^{\prime}\right)\right]$ is always injective, then the distance between successive iterates of $v$ and $v^{\prime}$ is persistently the same, and we must be able to interpose a Fatou periodic vertex $\omega$ in the middle in order not to violate the expanding condition. We now apply Lemma 6.4 to the two branches at $\omega$ and get a contradiction. 
Finally, if $v, v^{\prime}$ are not periodic, then thanks to Lemma 6.2 we transfer inductively the question to $f(v)$ and $f\left(v^{\prime}\right)$ until both vertices become periodic.

7. Fit extensions. In this section we define a class of extensions which adds nothing essential to a Hubbard tree. Their merit is to help us correctly draft potential Fatou components. As a by product, we show how to construct canonical extensions with all ends of Julia type. This standardization will prove useful later on.

Let $H$ be a Hubbard tree. An edge $e$ incident at a Fatou vertex $\omega$ is fit when its other endpoint $v$ is of Julia type and satisfies $\operatorname{dist}\left(f^{\circ n}(\omega), f^{\circ n}(v)\right)$ $=1$ for all $n$. In plain words, the edge is delineating an internal ray in the Böttcher coordinate. Likewise, a Fatou vertex is fit if all its incident edges are fit. Here we are shielding a Fatou component. A Hubbard tree is fit if all its Fatou vertices are fit. The purpose of this section is to establish the existence of canonical fitted extensions.

Lemma 7.1. Every Hubbard tree admits a canonical extension with all periodic Fatou vertices fit. In fact, the underlying topological trees can be chosen the same, with only new Julia vertices added.

Proof. Let $\omega$ be a periodic Fatou vertex and $e$ an edge with endpoints $\omega$ and $v$.

Suppose first that the germ of $e$ is periodic, say of period $k$. We distinguish two cases: when $\operatorname{dist}\left(f^{\circ m}(\omega), f^{\circ m}(v)\right)=1$ for all $m$, or not.

In the former case, following Lemma 5.2, all edges $e_{m}=\left[f^{\circ m}(\omega), f^{\circ m}(v)\right]$ are homeomorphic. Also, when $v$ is of Julia type, then this edge $e$ is already fit. If $v$ is of Fatou type, we insert a vertex $v_{m}$ in each $e_{m}$ (in the event of $e_{m}=e_{l}$, make sure to have $v_{m}=v_{l}$ even when $\left.f^{\circ m}(\omega) \neq f^{\circ l}(\omega)\right)$, and define $f\left(v_{m}\right)=v_{m+1}$. Then clearly $v_{0}$ is periodic of period $k$ or $k / 2$. The angles at $v_{k}$ are $1 / 2$ because two edges meet there. This gives an expanding tree since the only new vertices are the $v_{j}$, of Julia type, inserted between $f^{\circ j}(\omega)$ and $f^{\circ j}(v)$, both of Fatou type. The extension is canonical due to Proposition 5.6: inside $e_{m}$ there is place for at most one Julia vertex.

Otherwise we will have $\operatorname{dist}\left(f^{\circ m}(\omega), f^{\circ m}(v)\right)>1$ for some $m \geq 1$. If so, we insert different vertices $v_{j}$, one in each $e_{j}$, for $j=0, \ldots, k-1$, as close as possible to $\omega_{j}=f^{\circ j}(\omega)$ (this time $e_{j}=e_{j+k / 2}$ implies $v_{j} \neq v_{j+k / 2}$ ), and define $f\left(v_{j}\right)=v_{j+1}$. These points $v_{j}$ have exact period $k$. Again angles at $v_{k}$ measure $1 / 2$. Next we prove that this new abstract tree is expanding also at the new vertices. Suppose $\hat{v}$, of Julia type, is contiguous to $v_{j}$. This implies $\hat{v} \neq \omega_{j}$, simply because the types of $\omega_{j}$ and $\hat{v}$ are different. If $\operatorname{dist}_{\text {NEW }}\left(f^{\circ i}\left(v_{j}\right), f^{\circ i}(\hat{v})\right)=1$ for all $i$ (we are talking about the distance in the new tree), then $\operatorname{dist}_{\mathrm{NEW}}\left(f^{\circ i}\left(\omega_{j}\right), f^{\circ i}(\hat{v})\right)=2$, for all $i$, owing to the fact 
that no $f^{\circ i}\left(v_{j}\right)$ is critical. If $\hat{v}$ belongs to the old tree, the last relation in turn forces $\operatorname{dist}\left(f^{\circ i}\left(\omega_{j}\right), f^{\circ i}(\hat{v})\right)=1$ in the original tree, in contradiction to what was assumed earlier. Else $\hat{v}$ should be one of the new vertices. Now the same reasoning as above ensures that in the original tree the distance between the successive iterates of $\omega_{j}=f^{\circ j}(\omega)$ and $f^{\circ j}(v)$ - the endpoints of $e_{j}$, where by definition $v_{j}$ and $\hat{v}$ stand-will remain 1 . This is not the case.

To tackle the general case by induction, suppose the edge $f(e)$ is fit but $e$ is not. As we already know how to make fit periodic edges, we suppose $e$ is not periodic. Let $f(\omega)$ and $\hat{v}$ be the endpoints of $f(e)$, and $\omega$ and $v$ be those of $e$. By assumption $\hat{v}$ is of Julia type since $f(e)$ is fit. We also have $f(v) \neq \hat{v}$ (if not, $e$ is fit). Here we insert a vertex $\tilde{v}$ between $\omega$ and $v$, and define $f(\tilde{v})=\hat{v}$. Notice that $\tilde{v}$ does not belong to the forward orbit of $\hat{v}$ for otherwise it will already be present in the old tree, which does not happen. We copy the angles at $\tilde{v}$ from those at $\hat{v}$. This tree is expanding as no new periodic points are added. The extension is canonical since the restriction of $f$ to $e$ is injective and as such there is precisely one way to choose the preimage of a point modulo isotopy.

Lemma 7.2. Every Hubbard tree admits a canonical extension with all Fatou vertices fit. In fact, the underlying topological trees can be chosen to be the same, with only new Julia vertices added.

Proof. Start with an extension as in Lemma 7.1. Then inductively make fit any edge that stems from strictly preperiodic Fatou vertices using the same technique as at the end of the proof of the last lemma. We are never appending new periodic vertices so expansiveness is granted for free.

For purposes that will become apparent shortly, we need a small improvement in the quality of the extensions.

Proposition 7.3. Every Hubbard tree admits a canonical fit extension where every end is of Julia type.

Proof. We assume directly that our tree is fit. Suppose first that the Fatou vertex $\omega$ is an end but $f(\omega)$ is not. If so, let $e$ be the lone edge that stems from $\omega$. Take any edge $e_{1}$ other than $f(e)$ at $f(\omega)$. Let $v_{1}$ be the Julia vertex that is connected to $f(\omega)$ via $e_{1}$. Now extend the tree by adding a new edge $e_{0}$ with endpoints $\omega$ and $v_{0}$ making an angle of $\angle_{f(\omega)}\left(f(e), e_{1}\right) / d(\omega)$ with $e$-as usual $d(\omega)$ is the local degree at $\omega$. When we map $v_{0}$ to $v_{1}$, this fit extension is still expanding.

The only danger is if a periodic Fatou orbit is composed of ends. In such a case all edges stem at the zero argument in a suitable Böttcher coordinate. At a critical $\omega$ we append an edge at angle $1 / d(\omega)$ and make it fold under 
iteration. This critical point is no longer an end and we can repeat the work of the last paragraph.

8. Canonical inverse trees. We now describe an important class of canonical extensions. For the Hubbard tree $H_{f, M}$ generated by a polynomial $f$ and the invariant set $M$ we reconstruct the tree generated by $f^{-1} M$ using only $H_{f, M}$ as data. This section represents the abstract counterpart to Section 3.

A Hubbard tree of degree $d$ is homogeneous if every vertex with a preimage - in the vertex dynamics - has $d$ of them (counting multiplicity). In other words, one preferred inverse implies a full set (compare Corollary 6.3). A standard count gives the following.

Lemma 8.1. A degree $d$ Hubbard tree with set of vertices $V$ is homogeneous if and only if $|V|-1=d(|f(V)|-1)$. Here $|\cdot|$ stands for cardinality.

The term is justified by the fact that the underlying topological tree can be chopped into $d$ pieces, each homeomorphic as a graph to the tree generated by restriction to $f(V)$.

Lemma 8.2. Let $T^{*}$ be any partition (as in Section 6) of the homogeneous Hubbard tree $H$. Then each $T \in T^{*}$ is homeomorphic to $f(H)=[f(V)]$.

Proof. Writing $V_{T}=V \cap T$ we get $T=\left[V_{T}\right]$. Since the tree is homogeneous, we have $f\left(V_{T}\right)=f(V)$. Thus, the chain of inclusions

$$
[f(V)]=\left[f\left(V_{T}\right)\right] \subset f\left(\left[V_{T}\right]\right) \subset\left[f\left(V_{T} \cup \Omega\right)\right] \subset[f(V \cup \Omega)]=[f(V)]
$$

guarantees that $f$ maps $T=\left[V_{T}\right]$ onto $[f(V)]$. As $f$ restricted to $T$ is injective, the result follows.

Let $H^{\prime}$ be a homogeneous extension of $H$. If $f\left(V_{H^{\prime}}\right)=V_{H}$, we say that $H^{\prime}$ is the inverse of $H$ and write $\operatorname{inv}(H)=H^{\prime}$. In fact, inverses are unique.

Proposition 8.3. Every Hubbard tree admits a unique inverse.

We organize the proof in several stages. While attacking uniqueness through necessary conditions, we will also hint how to handle existence.

Proof of uniqueness. Take two inverses of $H$. Around each critical vertex in $H$ select an edge as reference. In a synchronized manner chop both inverses as in Section 6. According to Lemma 8.2, every resulting piece is homeomorphic to $H$. After matching corresponding pieces, we find no structural difference between one set of subtrees and the other. (Around the critical vertices we must be cautious about angles. However the presence of a common reference edge in all three trees allows coherence.)

EXAMPLE 8.4. We will reconstruct from scratch the inverse of the tree presented in Example 6.1. According to the method just outlined, near each 
critical vertex we pick a reference edge: we grab the ones we choose before, in Section 6. Before proceeding further, we construct an intermediate extension $\hat{H}$. As the segment $A B$ is somehow marked, we travel half a turn to find an empty spot. We append precisely there a segment $A B^{\prime}$ where $B^{\prime}$ maps to $D$, like $B$. Notice that the segments $A B^{\prime}$ and $A B$ fold under iteration. Furthermore, as $B^{\prime}$ is preperiodic, the new tree remains expanding. Near $C$ the preferred arc $C D$ takes $C B$ as a folding companion (here it is irrelevant whether or not $B$ and $D$ map to the same point); we take no further action. Finally, at $D$ the preferred direction is $D C$. Since the edge pointing at angle $1 / 2=1 / \operatorname{deg}(D)$ is missing, we append $D C^{\prime}$ and map $C^{\prime}$ to $E=f(C)$. The dynamics in this intermediate tree $\hat{H}$ is still expanding. As we will confirm briefly, these newly added segments play the role of mounting arms where we will assemble subtrees to get the full inverse. This tree $H^{\prime}$ is the one we subdivide as in Section 6 into $T_{1}, T_{2}, T_{3}, T_{4}$.

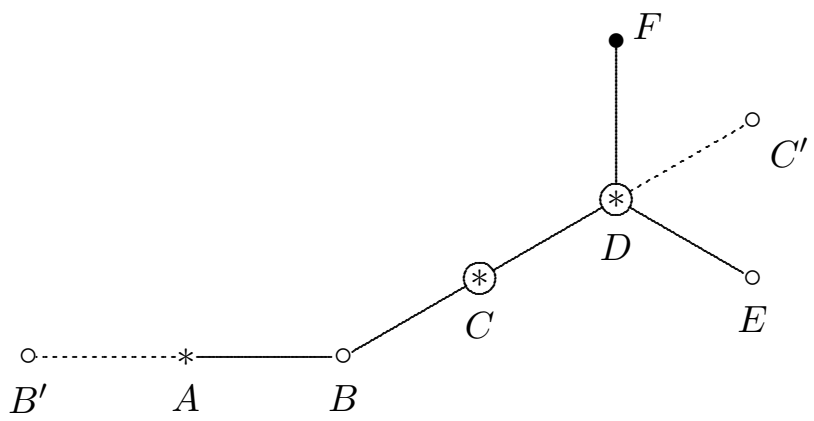

Fig. 8.1

Now we think of each $T_{j}$, for $j=1, \ldots, 4$, as mapping into a different copy $H_{j}$ of the original tree $H$. In this way, a vertex in $T_{j}$ rightfully deserves the name $f_{j}^{-1}(v)$, where $v$ is a vertex in $H$. We can even use $v_{j}^{-1}$ as shorthand to make figures tractable.

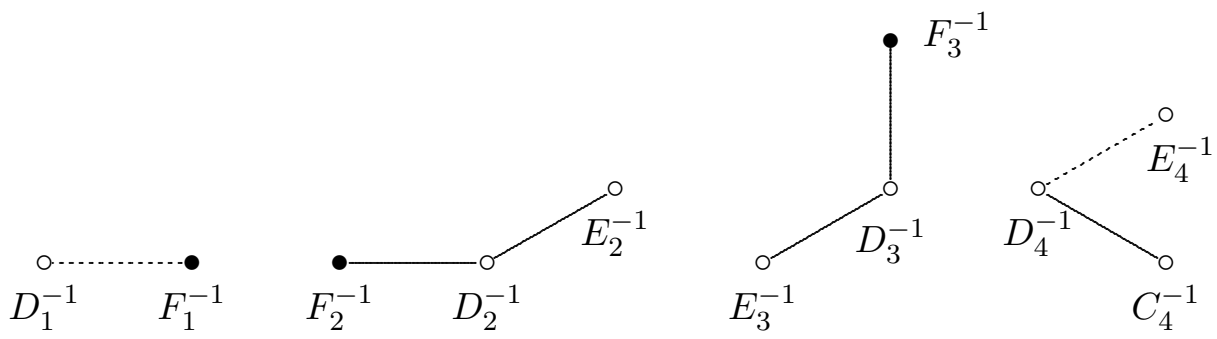

Fig. 8.2. This is Figure 6.3 after relabeling vertices and appending virtual edges.

The original map $f$ is injective when restricted to each piece. Hence, we can embed $T_{j}$ into a different copy $H_{j}$ of $H$ and identify each point with its 
image. In practice, we are filling empty spaces. The subindex $j$ will help us recall the background.
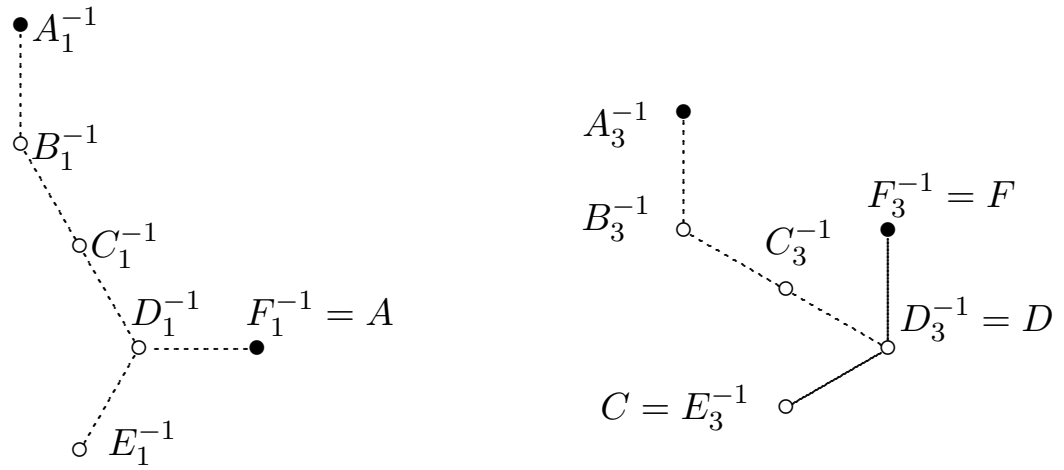

Fig. 8.3. How the first and third subtrees are immersed in the inverse. Do not forget the equalities $F_{1}^{-1}=A, E_{3}^{-1}=C, D_{3}^{-1}=D, F_{3}^{-1}=F$.

Regarding Figure 8.3, for the piece on the left everything looks fine. However, at first glance something is wrong with the one on the right: the displayed angles around $D=D_{3}^{-1}$ do not match those around $D$ in $H$, the raw model. Even if this is not conceptually incorrect (Lemma 6.2 promises only a topological equivalence with the image), corrective measures will be enforced.

About a critical point $\omega$ angles get multiplied by $d(\omega)$. That is why to keep matters injective, we restrict to sectors of span $1 / d(\omega)$ starting from the benchmark. Therefore, around critical points the picture ought to get squeezed. This explains visual inconsistencies. Let us work tree by tree.

In the first tree the only critical point is $A$, recognized in the picture as $F_{1}^{-1}$. The reference segment, even if only of virtual nature, is $A B^{\prime}$, and here it appears as $F_{1}^{-1} D_{1}^{-1}$. However, in the first tree there is no other segment incident at $A$, so there are no angles to rectify.

The second tree requires some care as there are two critical points: $A=$ $F_{2}^{-1}$ with preferred direction $A B=F_{2}^{-1} D_{2}^{-1}$ and $C=E_{2}^{-1}$ with preferred direction $C B=E_{2}^{-1} D_{2}^{-1}$. Fortunately, there are no other edges in the extended tree incident at any of these two dangerous points, and there is nothing to fix.

In the third tree the critical vertices are $C, D$-depicted as $E_{3}^{-1}, D_{3}^{-1}$, based at $C D$ and $D C$, respectively. Therefore, taking $D F=D_{3}^{-1} F_{3}^{-1}$ as reference, near $D=D_{3}^{-1}$ the angles should be halved. [Recall that edges are recollected finishing at $D C$, so $D F$ is more vulnerable to changes.] No action is taken close to $C=E_{3}^{-1}$ as we see only one branch.

In the last tree, only $D_{4}^{-1}=D$ is critical. The tree must fit inside a wedge of 180 degrees finishing at the virtual - yet distinguished - direction $D C^{\prime}=D_{4}^{-1} E_{4}^{-1}$. 

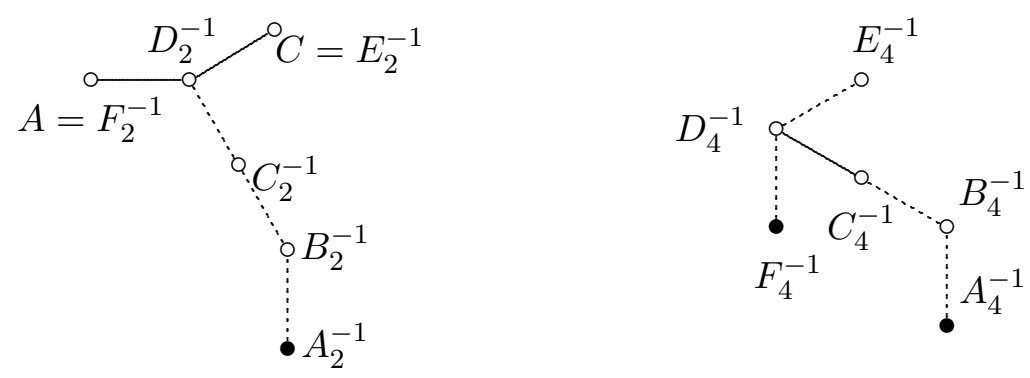

Fig. 8.4. The second and fourth subtrees in full

Having now the four homeomorphic images of $H$ (not of $\hat{H}$ !), we mount them upon the original skeleton to get the inverse tree. Of course, a point of the form $v_{j}^{-1}$ maps to $v$ under $f$.

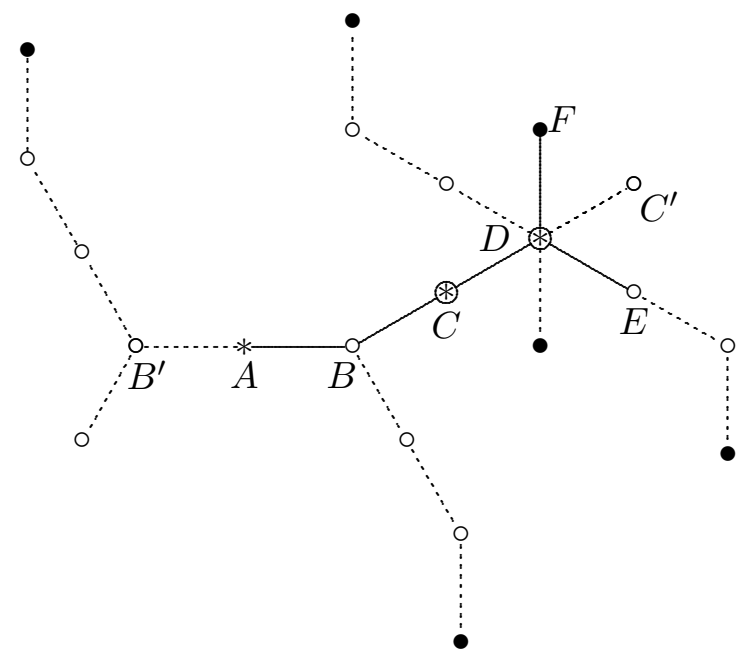

Fig. 8.5. The inverse tree

Proof of existence. As suggested by the example, we first build an intermediate tree $\hat{H}$. As usual, at every critical point $\omega$ we pick a preferred edge $e=e_{\omega}$. If we find no edge making an angle of $k / d(\omega)$ turns with $e$, then we insert a copy $e_{k}$ of $e$ there. With the obvious dynamics $f(e)=f\left(e_{k}\right)$ we still have a well behaved angled tree. As no new periodic points are added, the expanding condition is satisfied. Once this precaution is taken at all critical places, call the resulting expanding tree $\hat{H}$. This tree must be differentiated from $H$ since it is probably bigger.

Next we subdivide $\hat{H}$ as in Section 6. Call the resulting family $T^{*}$. Now, each $T_{j} \in T^{*}$ is mapped injectively into $f(\hat{H})=f(H)$ as indicated in Lemma 6.2. Consider different copies $H_{j}$ of $H$ and embed $T_{j}$ in $H_{j}$ following $f$ (cf. Example 8.4). Calibrate the angles at the critical vertices as 
in Example 8.4. Once we reglue all $H_{j}$ on top of $\hat{H}$, we have a new simplicial complex with well defined angles. If we map each point in $H_{j}$ to the actual point from $H$ from which it was cropped, we get a well defined dynamics compatible with the angles. Also, an Euler characteristic argument shows that this new connected graph is a tree, clearly the inverse tree. As no new periodic vertices are added, the tree is expanding. By Lemma 8.1 this Hubbard tree is homogeneous.

THEOREM 8.5. The inverse is a canonical extension.

Proof. If $H^{\prime}$ is an extension of $H$, we construct the inverse of $H^{\prime}$ as in the last theorem. This is a common extension of $\operatorname{inv}(H)$ and $H^{\prime}$. Indeed, in $\operatorname{inv}\left(H^{\prime}\right)$ we restrict to $\left[f^{-1}(V)\right]$, where $V$ is the original collection of vertices. This restriction tree should be $\operatorname{inv}(H)$ due to uniqueness.

This is the correct moment to review the concept of incidence. We now carefully study from the dynamical point of view how the number of edges incident at a Julia vertex grows with the inverse.

Let $H$ be a Hubbard tree. We define the incidence number $\nu_{H}(v)$ at $v \in V$ as the number of connected components of $T-\{v\}$ in the underlying topological tree. In the successive inverse trees $\operatorname{inv}^{\circ m}(H)$, we also have incidence functions $\nu_{H, m}=\nu_{\mathrm{inv}}^{\circ m}(H)$. By definition we have $\nu_{H, 0}(v) \leq \nu_{H, 1}(v)$ whenever $v \in V$. Also, by construction of $\operatorname{inv}(H)$ we get $\nu_{H, 1}(v)=d(v) \nu_{H, 0}(f(v))$ for $v \in \operatorname{inv}(H)$.

Proposition 8.6. For every periodic Julia vertex $v \in V$ and integer $m \geq 0$ we have $\nu_{H, 0}(v)=\nu_{H, m}(v)$.

Proof. Even if this fact is engulfed by Proposition 5.11, we present a constructive argument. As $d\left(v^{\prime}\right)=1$ at every $v^{\prime} \in \mathcal{O}(v)$, no new edges are added around $v$ in the construction of $\operatorname{inv}^{\circ m}(H)$.

COROLlary 8.7. Let $v \in V$ be a Julia vertex such that $f^{\circ k}(v)$ is periodic. Then for every $m \geq k$ we have $\nu_{H, k}(v)=\nu_{H, m}(v)$.

COROLlary 8.8. Let $H$ be a Hubbard tree. There is a $k \geq 0$ such that for all $m \geq k$ we have $\nu_{H, k}(v)=\nu_{H, m}(v)$ at every original Julia vertex $v \in V_{H}$.

Proof. In fact, there are only a finite number of vertices in the original tree.

We denote such numbers by $\nu_{H, \infty}(v)$.

9. Accesses and external coordinates. We now associate an angle to every Julia based access. In practice this value represents the argument of a landing ray.

Let us focus on the disposition around a vertex $v \in V$. If $e, e^{\prime}$ are consecutive edges next to $v$, then the triplet $\mathcal{A}=\left(v, e, e^{\prime}\right)$ is called the pseudoaccess 
based at $v$ and supported by $e$. A vertex can be base for multiple pseudoacesses. On the other hand, an edge supports exactly two pseudoaccesses: one for each endpoint.

The edge $e^{\prime}$ has endpoints $v$ and some other vertex $v^{\prime} \in V$. When $e^{\prime \prime}$ follows $e^{\prime}$ around $v^{\prime}$, the pseudoaccess $\mathcal{A}^{\prime}=\left(v^{\prime}, e^{\prime}, e^{\prime \prime}\right)$ becomes the successor of $\mathcal{A}=\left(v, e, e^{\prime}\right)$ (in the pseudoaccess order). This reflects the idea that a pseudoaccess is barely a way to flip from one edge to another using the vertex as hinge.
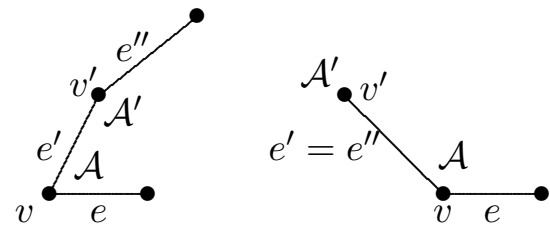

Fig. 9.1. A glimpse at the pseudoaccesses

Of course, when we leap from vertex to vertex around the tree, few surprises await us. For example, the next properties are somehow trivial.

LEMma 9.1. In a Hubbard tree the pseudoaccesses are arranged in cyclic order.

Proof. A trivial induction on the size of $V$, the set of vertices.

Two subsets $\mathcal{T}, \mathcal{S}$ of the circle are unlinked if they belong to disjoint connected subsets of $\mathbb{T}$, or, equivalently, if $\mathcal{S}$ is contained in one component of $\mathbb{T}-\mathcal{T}$. In particular, these sets are disjoint. If we identify $\mathbb{T}$ with the boundary of the unit disk, an analogous condition is that the convex hulls of $\mathcal{T}$ and of $\mathcal{S}$ are disjoint.

Lemma 9.2. Given $p \in H$, pseudoaccesses based at points belonging to different components of $H-\{p\}$ are pairwise unlinked.

Lemma 9.3. Arrange the edges at $v$ as $e_{0}, \ldots e_{m}=e_{0}$. Let $\mathcal{A}_{i}$ be any pseudoaccess whose supporting edge belongs to $\mathcal{B}_{v}\left(e_{i}\right)$, the branch that stems from $e_{i}$. Then $\mathcal{A}_{0}<\mathcal{A}_{1}<\cdots<\mathcal{A}_{0}$ in the cyclic order.

Fix a decomposition $T^{*}$ of $H$ as in Section 6. When $e$ is contained in $T \in T^{*}$, by some abuse of language we even say that the access $\mathcal{A}=\left(v, e, e^{\prime}\right)$ is supported at $T$.

Lemma 9.4. Pseudoaccesses supported at different pieces of $T^{*}$ are pairwise unlinked.

Proof. Let $T_{1}, T_{2}$ be different members of $T^{*}$. If $T_{1} \cap T_{2}=\emptyset$, we find $p$ so that $T_{1}, T_{2}$ are included in different components of $H-\{p\}$. This case is then settled by Lemma 9.2. Otherwise $T_{1}$ and $T_{2}$ intersect at a critical 
point $v$. We define $\hat{T}_{i}$ as the union of branches that stem from $v$ and contain an edge in $T_{i}$. Now we get more than we asked for: a pair of pseudoaccesses supported in $\hat{T}_{1}$ is unlinked from a pair supported at $\hat{T}_{2}$ due to Lemma 9.3, because each bouquet is the union of cyclically arranged branches.

A pseudoaccess $\left(v, e, e^{\prime}\right)$ becomes an access if $\nu_{H, 0}(v)=\nu_{H, \infty}(v)$, that is, if the number of edges around $v$ is stable. By definition, at Fatou vertices there are no possible accesses whatsoever since the incidence keeps growing with the inverses. Clearly, an access at $v$ is periodic if and only if $v$ is periodic and of Julia type. In what follows, whenever $\mathcal{A}$ is an access (or pseudoaccess), we write $v_{\mathcal{A}}$ for the base point.

Lemma 9.5. Let $H$ be a Hubbard tree of degree $d$. Then $f$ induces a degree $d$ orientation preserving covering mapping between the pseudoaccesses of $\operatorname{inv}(H)$ and $H$. Furthermore, accesses in $\operatorname{inv}(H)$ map to accesses in $H$.

Proof. If $\left(v, e, e^{\prime}\right)$ is a pseudoaccess $\operatorname{in} \operatorname{inv}(H)$, then $\left(f(v), f(e), f\left(e^{\prime}\right)\right)$ is by construction a pseudoaccess in $H$. Clearly the correspondence is $d$-to- 1 and order preserving. The second part is obvious.

REMARK 9.6. For the access $\mathcal{A}=\left(v, e, e^{\prime}\right)$ there is a mild psychological preponderance of $e$ over $e^{\prime}$. This is a consequence of fixing an orientation. (In fact, in the mirror Hubbard tree, the access $\mathcal{A}$ corresponds to $\overline{\mathcal{A}}=\left(\bar{v}, \bar{e}^{\prime}, \bar{e}\right)$ and successor becomes predecessor.)

Now we are ready to build a bridge between the theory of Hubbard trees and the dual theory of critical portraits. The angle associated to an access at the end will be that of a landing ray.

Proposition 9.7. Let $H$ be a homogeneous Hubbard tree of degree $d>1$ with at least one Julia vertex. There exist an embedding $\phi_{H}$ of the accesses of $H$ into $\mathbb{T}$ for which the induced map between accesses becomes multiplication by $d$ (modulo 1). Furthermore, $\phi_{H}$ is uniquely defined up to a global addition of a multiple of $1 /(d-1)$.

Proof. In order to assign an argument to each access of $H$, we can work in a larger tree $\operatorname{inv}^{\circ m}(H)$, where $m$ is large enough. Even more, we assume without loss of generality that every end is of Julia type (compare Proposition 7.3). We still call this tree $H$.

By Lemma 9.5 the induced map between accesses is an orientation preserving covering of degree $d$. To assign an argument we prove that this finite map is expanding (compare the first appendix in Section 14). Take in $H$ two adjacent periodic accesses $\mathcal{A}_{i}=\left(v_{i}, e_{i}, e_{i}^{\prime}\right), i=0,1$. The idea is to show that for some $m$ large enough these accesses cannot be consecutive in inv ${ }^{\circ m}(H)$. As no new periodic Julia vertices are added in the construction of inv ${ }^{\circ m}(H)$, no new periodic accesses appear and the conditions of Theorem 14.4 are triv- 
ially met; this will establish the result. We distinguish between $v_{0}=v_{1}$ and $v_{0} \neq v_{1}$.

If $v_{0}=v_{1}$, then part of the cyclic string around this vertex is $e_{0}<e_{0}^{\prime} \leq$ $e_{1}<e_{1}^{\prime} \leq e_{0}$ (actually $e_{0}^{\prime}=e_{1}$, but this is irrelevant). Due to Lemma 9.3, it is enough to find $m \geq 0$ so that inv ${ }^{\circ m}(H)$ allows an access inside the branch $\mathcal{B}_{v_{0}, \text { inv }^{\circ m}(H)}\left(e_{0}^{\prime}\right)$. But this is easy. As every end of the tree is of Julia type, one of them already belongs to $\mathcal{B}_{v_{0}, H}\left(e_{0}^{\prime}\right)$. Now we apply Corollary 8.7 to make sure there is an access in place.

Now suppose $v_{0}, v_{1}$ are different periodic Julia points. For some $m>0$ there is a vertex $v^{\prime} \in \operatorname{inv}^{\circ m}(H)$ inside the segment $\left[v_{0}, v_{1}\right]$ for otherwise our Hubbard tree will not be expanding. If $v^{\prime}$ is of Julia type, we grow the tree until all pseudoaccesses at $v^{\prime}$ turn accesses. Then $\mathcal{A}$ and $\mathcal{A}^{\prime}$ are no longer consecutive in the cyclic arrangement. Else, based at the Fatou vertex $v^{\prime} \in \operatorname{inv}^{\circ m}(H)$, let $\left(v^{\prime}, e, e^{\prime}\right)$ be a pseudoaccess between $\mathcal{A}_{0}, \mathcal{A}_{1}$ in the cyclic order. Find an argument $\theta$ between $\varphi(e)$ and $\varphi\left(e^{\prime}\right)$ (in the Böttcher coordinate $\varphi$ at $v^{\prime}$ ) which via multiplication by the step by step degrees of the Fatou critical points along the orbit of $v^{\prime}$ catches up with either $\varphi(e)$ or $\varphi\left(e^{\prime}\right)$. Then eventually (that is, in some appropriate inverse tree) a branch will stem from this direction. As all ends should be of Julia type, we can work as before to grow an access on the said branch.

In all cases we have proved that between periodic accesses we can interpolate a nonperiodic one. In this way the map is shown to be expanding. By Theorem 14.4, it can be angled precisely in $d-1$ ways.

As every abstract Hubbard tree $H$ has a canonical extension satisfying the conditions of Proposition 9.7, we can associate to every access a coordinate compatible with the dynamics. No secret that such external coordinate $\phi_{H}$ will be a Böttcher coordinate in the basin of attraction of $\infty$.

Lemma 9.8. In some inverse $\operatorname{inv}^{\circ m}(H)$, at all Julia vertices of the original tree pseudoaccesses become accesses.

Proof. This is a way to rephrase Corollary 8.8.

Now let $\theta \mapsto d \theta \mapsto \cdots \mapsto d^{k} \theta=\theta$, be a periodic orbit under the standard $d$-fold multiplication in $\mathbb{T}$. An important question is whether there is a canonical extension of $H$ where the accesses corresponding to $\left\{\theta, d \theta, \ldots, d^{k-1} \theta\right\}$ are present. For this we have the following.

Proposition 9.9. Let $H$ be a homogeneous abstract Hubbard tree with at least one Julia vertex. For any choice of external coordinate $\phi_{H}$ and periodic orbit $\theta \mapsto d \theta \mapsto \cdots \mapsto d^{k} \theta=\theta$ under $d$-fold multiplication in $\mathbb{T}$, there is a canonical extension of $H$ in which the accesses corresponding to $\left\{\theta, d \theta, \ldots, d^{k-1} \theta\right\}$ are present. 
We defer the proof until the end. In fact, Section 13 is entirely devoted to it. For the time being we take this fact for granted and move speedily toward the realization of Hubbard trees.

10. From Hubbard trees to formal critical portraits. Now we employ canonical extensions to mimic the construction associated with critical portraits. The main concepts and results of this theory are reviewed in the second appendix of Section 14.

Every Hubbard tree admits a canonical fit extension. In it, given a Fatou vertex $\omega$ and an edge $e$ with endpoints $\omega$ and $v$, the vertex $v$ is of Julia type and the distance between iterates of $\omega$ and $v$ is persistently 1 .

Around a Fatou periodic orbit consider a system of Böttcher coordinates. About this critical cycle we extend the tree by appending an edge and a vertex at every zero argument (if they are not already present). This kind of extensions is canonical since either the periodic points are already in place or, else, we are just pasting ends contiguous to Fatou points. For any other Fatou vertex, say $w$, we suppose that the zero segment is present in the local chart for $f(w)$ and proceed by backward induction: we insert a new vertex and a new edge as preimages of the ones just given above. Since this last construction can also be worked out by restriction in the inverse tree, we still move in canonical territory. Near a Fatou vertex, the appearance of a preferred "natural" edge will make our lives easier when the time comes to split the tree according to the methodology introduced in Section 6 .

Corollary 8.8 guarantees that pseudoaccesses defined at the endpoints of all such zero internal rays are accesses in a suitable inverse. Any extension satisfying that condition is supporting.

Let $\omega$ be a Fatou vertex. An access $(v, \tilde{e}, e)$ is said to support $\omega$ if the endpoints of $e$ are $v, \omega$ and for all $k \geq 0$ they obey $\operatorname{dist}_{H}\left(f^{\circ k}(v), f^{\circ k}(\omega)\right)=1$. This is another way of saying that $e$ is fit, yet this time with emphasis on the access. Clearly $f(v, \tilde{e}, e)=(f(v), f(\tilde{e}), f(e))$ supports $f(\omega)$. When $(v, \tilde{e}, e)$ supports $\omega$ we rewrite it as $\mathcal{D}(\omega, e)$. (Compare Figure 10.1.)

Let $H$ be a supporting abstract Hubbard tree. Applying Corollary 8.8 we pick a suitable inverse $\operatorname{inv}^{\circ m}(H)$ in which at every original Julia vertex $v \in V_{H}$ the incidence is already stable: for them we have $\nu_{H, m}(v)=\nu_{H, \infty}(v)$. We list the Fatou and the Julia critical points as $\omega_{1}, \ldots, \omega_{l}$ and $c_{1}, \ldots, c_{k}$, respectively. Now we are ready to select hierarchic accesses as in the appendix.

Let $\omega$ be a periodic Fatou vertex. If $e_{0}$ is the edge with Böttcher argument 0 , then $\mathcal{D}\left(\omega, e_{0}\right)$ has the same period as $\omega$. Map it as $f\left(\mathcal{D}\left(\omega, e_{0}\right)\right)$ and then pull it back to get $d(\omega)$ supporting accesses at $\omega$ (work in the inverse tree if you wish); of course, the original $\mathcal{D}\left(\omega, e_{0}\right)$ is among them. Collect them together in the "preargument" set $\mathcal{F}_{\omega}$. As the choice of a preferred edge is compatible along the cycle, the selection of preferred accesses is hierarchic. 
For nonperiodic Fatou critical points, again denote by $\mathcal{D}\left(\omega, e_{0}\right)$ the access that hangs from the zero internal segment. Push it forward and then pull it back to form $\mathcal{F}_{\omega}$, a set with $d(\omega)$ elements.

For a Julia critical vertex $c$ we start the closest to a periodic orbit. Find an access based at $f(c)$ and pull it back. Keep record of those $d(c)$ accesses as $\mathcal{J}_{c}$. Choose a hierarchic element in the way. For future reference, the edge where the original access is supported will be the preferred edge around $c$. Continue inductively.

There is a slight difference in the two constructions: at a Julia vertex the marked accesses are based at the critical point, while for Fatou vertices the accesses are taken at the other end of the preferred internal edge.

In this way, we have constructed two families

$$
\mathcal{F}=\left\{\mathcal{F}_{\omega_{1}}, \ldots, \mathcal{F}_{\omega_{l}}\right\}, \quad \mathcal{J}=\left\{\mathcal{J}_{c_{1}}, \ldots, \mathcal{J}_{c_{k}}\right\}
$$

of accesses. We will not distinguish between the access itself and the corresponding external coordinate assigned by $\phi_{H}$ (compare Proposition 9.7).

The following is a trivial consequence of all we have done.

Proposition 10.1. The marking $\Theta=(\mathcal{F}, \mathcal{J})$ represents a formal critical portrait.

Of course, we still have to verify if this critical portrait is admissible. Having set out goals, here are two easy properties we want to isolate. Their purpose is to place in $\mathbb{T}$ a given access (the argument associated to it, really) relative to the preferred sets $\mathcal{F}_{\omega}, \mathcal{J}_{c}$. Remember we are working in a supporting tree.

Lemma 10.2. Given a Fatou critical point $\omega$ of local degree $n>1$, write $e_{i}$ for the edge that stems from the internal angle of value $i / n$, so that $\mathcal{F}_{\omega}=\left\{\mathcal{D}\left(\omega, e_{0}\right), \ldots, \mathcal{D}\left(\omega, e_{n-1}\right)\right\}$ is displayed in cyclic order. Suppose $\mathcal{C}$ is an access supported in the branch $\mathcal{B}_{\omega}(e)$. If $e_{i-1}<e \leq e_{i}$ (in the cyclic order around $\omega)$, then $\mathcal{D}\left(\omega, e_{i-1}\right)<\mathcal{C} \leq \mathcal{D}\left(\omega, e_{i}\right)$.

Proof. If $e_{i-1}<e<e_{i}$, then Lemma 9.3 gives directly $\mathcal{D}\left(\omega, e^{\prime}\right)<\mathcal{C}<$ $\mathcal{D}(\omega, e)<\mathcal{D}\left(\omega, e^{\prime}\right)$. Else we have $e=e_{i}$ and we are just capturing the idea that $\mathcal{D}\left(\omega, e_{i}\right)$ is the last among all accesses supported at $\mathcal{B}_{\omega}\left(e_{i}\right)$.

Lemma 10.3. Let $c$ be a Julia type critical point of degree $n$. Write $\mathcal{J}_{c}=$ $\left\{\mathcal{A}_{0}, \ldots, \mathcal{A}_{n-1}\right\}$, where $\mathcal{A}_{i}=\left(c, e_{i}, e_{i}^{\prime}\right)$, in such a way that $e_{0}<e_{1}<\cdots<$ $e_{n}=e_{0}$ reflects the cyclic order of the supporting edges. Suppose $\mathcal{C}$ is an accesses supported in a branch $\mathcal{B}_{c}(e)$. If $e_{i-1}<e \leq e_{i}$, then $\mathcal{A}_{i-1}<\mathcal{C} \leq \mathcal{A}_{i}$.

Proof. This is the Julia-like version of Lemma 10.2.

The choice of a preferred access related to a Fatou or a Julia point is equivalent to the choice of a preferred edge incident at the said critical point. 


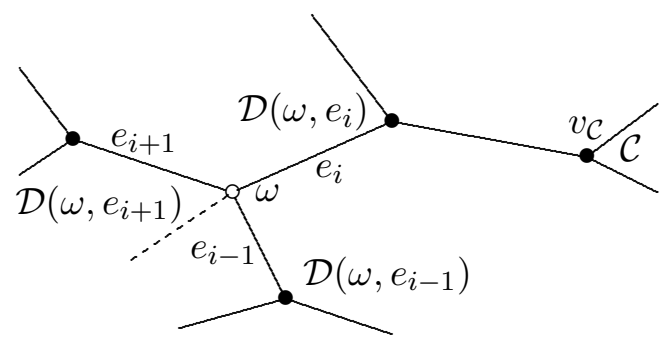

Fig. 10.1. Lemma 10.2 at work. Obligatorily we have $v_{\mathcal{C}} \neq \omega$ as one is a Fatou vertex while the other is of Julia type.

Near a Fatou critical point the edges in the zero direction are taken, while at Julia critical points we have the supporting edge of the preferred access. This is just the information we needed in Section 6 to construct a family of subtrees $T^{*}$. As a consequence, a supporting Hubbard tree automatically comes with a partition $T^{*}$.

In Section 6 we made the apparently unnatural decision to pick edges clockwise in the construction of $T^{*}$. This was done in order to make useful Lemmas 10.2 and 10.3. For example, they will render obvious Lemma 11.7.

11. From Hubbard trees to admissible critical portraits. Here we confirm the admissibility of the formal critical portrait constructed in the last section. For this we verify conditions (c6) and (c7) from Section 14. We first attack (c6). While we check (c7), the intermediate steps will help us show that any polynomial with critical marking $(\mathcal{F}, \mathcal{J})$ has Hubbard tree equivalent to the starting one. This is how Theorem 1.1 gets established.

In a sense, we only have to mimic the theory of critical portraits. Therefore, to fully understand this section, it is helpful to be familiar with addresses and symbol sequences. We will freely use the notation in Section 14. The goal is to prove that the formal critical portrait $\Theta=(\mathcal{F}, \mathcal{J})$ constructed so far is admissible.

Lemma 11.1. Let $\mathcal{A}_{i}, \mathcal{B}_{i}$ be accesses based at $v_{i}$ for $i=1$, 2. If $v_{1} \neq v_{2}$, then $\left\{\mathcal{A}_{1}, \mathcal{B}_{1}\right\}$ and $\left\{\mathcal{A}_{2}, \mathcal{B}_{2}\right\}$ are unlinked.

Proof. Let $p$ be any point between $v_{1}$ and $v_{2}$. Then $\left\{\mathcal{A}_{i}, \mathcal{B}_{i}\right\}$ are located at different sides of $T-\{p\}$ and we can apply Lemma 9.2.

First we study right symbol sequences. Fortunately they only have relevance for periodic arguments. We isolate some trivial facts.

Lemma 11.2. Let $\mathcal{A}, \mathcal{A}^{\prime}$ be distinct periodic accesses. If for some $k \geq 0$ we can find a Julia type critical point in the segment $\left[f^{\circ k}\left(v_{\mathcal{A}}\right), f^{\circ k}\left(v_{\mathcal{A}^{\prime}}\right)\right]$, then $s_{\Theta}^{+}(\mathcal{A}) \neq s_{\Theta}^{+}\left(\mathcal{A}^{\prime}\right)$. 
Proof. As $v_{\mathcal{A}}, v_{\mathcal{A}^{\prime}}$ are periodic and of Julia type, none of their iterates is critical. If a Julia critical point $c$ interposes between $f^{\circ k}\left(v_{\mathcal{A}}\right)$ and $f^{\circ k}\left(v_{\mathcal{A}^{\prime}}\right)$, then according to Lemma 10.3 the accesses $f^{\circ k}(\mathcal{A})$ and $f^{\circ k}\left(\mathcal{A}^{\prime}\right)$ belong to different components of $\mathbb{T}-\mathcal{J}_{c}$. The $k$ th right addresses of the initial accesses are then different.

Lemma 11.3. Let $\mathcal{A}=\mathcal{D}\left(\omega, e_{0}\right)$ be the periodic supporting access associated with the periodic Fatou critical point $\omega$. Suppose $\mathcal{A}^{\prime}$ is supported in a branch $\mathcal{B}_{\omega}\left(e^{\prime}\right)$ where $\angle_{\omega}\left(e_{0}, e^{\prime}\right)>1 / d(\omega)$. Then $\mathcal{A}$ and $\mathcal{A}^{\prime}$ have different right address.

Proof. Write $\mathcal{F}_{\omega}=\left\{\mathcal{D}\left(\omega, e_{i}\right)\right\}$ with the edges $e_{i}$ ordered as $e_{0}<e_{1}<$ $\cdots<e_{d(\omega)}=e_{0}$. Then $e_{1}<e<e_{0}$ implies $\mathcal{D}\left(\omega, e_{1}\right)<\mathcal{A}^{\prime}<\mathcal{D}\left(\omega, e_{0}\right)$ in view of Lemma 10.2. From this we get $\mathcal{D}\left(\omega, e_{0}\right)=\mathcal{A}<\mathcal{A}+\epsilon<\mathcal{D}\left(\omega, e_{1}\right)$ and the desired conclusion.

Lemma 11.4. Let $\mathcal{A}, \mathcal{A}^{\prime}$ be periodic accesses. If $s_{\Theta}^{+}(\mathcal{A})=s_{\Theta}^{+}\left(\mathcal{A}^{\prime}\right)$, then $v_{\mathcal{A}}=v_{\mathcal{A}^{\prime}}$.

Proof. By contradiction suppose $v_{\mathcal{A}} \neq v_{\mathcal{A}^{\prime}}$. As both vertices are periodic, we have $f^{\circ n}\left(v_{\mathcal{A}}\right) \neq f^{\circ n}\left(v_{\mathcal{A}^{\prime}}\right)$ for all $n$. The expanding condition (compare Proposition 5.6) guarantees that sooner or later we will find a critical point interior to $\left[f^{\circ k}\left(v_{\mathcal{A}}\right), f^{\circ k}\left(v_{\mathcal{A}^{\prime}}\right)\right]$. According to Lemma 11.2, it cannot be of Julia type. There are several routine cases to handle.

First suppose that $f$ restricted to all such intervals is injective. Then the number of vertices inside these segments is persistently the same, and all are periodic. With Proposition 6.5 at hand we can suppose further that $v_{\mathcal{A}}, v_{\mathcal{A}^{\prime}}$ belong to different pieces of the canonical decomposition $T^{*}$. Therefore at a Fatou critical point $\omega \in\left[v_{\mathcal{A}}, v_{\mathcal{A}^{\prime}}\right]$ we find different edges $e, e^{\prime}$ pointing to $v_{\mathcal{A}}, v_{\mathcal{A}^{\prime}}$. They have periodic endpoints, and thus correspond to periodic internal angles in the Böttcher coordinate. When we write $e_{0}, e_{1}, \ldots, e_{d(\omega)-1}$ for the preferred supporting edges at $\omega$, just one, say $e_{0}$, is periodic. If $e, e^{\prime}$ are both different from $e_{0}$, then they are intercalated in between $e_{0}, \ldots, e_{d-1}, e_{d}=e_{0}$. Then Lemma 9.3 implies directly that $\mathcal{A}$ and $\mathcal{A}^{\prime}$ are in different components of $\mathbb{T}-\mathcal{F}_{\omega}$. As a consequence, they do not share right address. However, if for instance $e=e_{0}$, then we must have $\angle\left(e^{\prime}, e_{0}\right) \geq 1 / d(\omega)$ (since $e=e_{0}$ and $e^{\prime}$ must belong to different members of $\left.T^{*}\right)$. Also $e^{\prime}$ is periodic, fact that rules out the equality $\angle\left(e^{\prime}, e_{0}\right)=1 / d(\omega)$. We get $e_{0}<e^{\prime}<e_{d(\omega)-1}$ and along we conclude $\mathcal{D}\left(\omega, e_{0}\right)<\mathcal{A}^{\prime}<\mathcal{D}\left(\omega, e_{d(\omega)-1}\right)<\mathcal{A} \leq \mathcal{D}\left(\omega, e_{0}\right)$. Hence, the only way to still have a slight chance of matching right address is to allow $\mathcal{A}=\mathcal{D}\left(\omega, e_{0}\right)$. But $f$ is injective in $\left[f^{\circ n}\left(v_{\mathcal{A}}\right), f^{\circ n}\left(v_{\mathcal{A}^{\prime}}\right)\right]$. We conclude that $f^{\circ n}\left(v_{\mathcal{A}^{\prime}}\right)$ belongs to $\mathcal{B}_{f^{\circ n}(\omega)}\left(f^{\circ n}\left(e^{\prime}\right)\right)$, the branch that stems from $f^{\circ n}(\omega)$ through the $f^{\circ n}\left(e^{\prime}\right)$ edge. Furthermore, we have $f^{\circ n}(\mathcal{A})=\mathcal{D}\left(f^{\circ n}(\omega), f^{\circ n}\left(e_{0}\right)\right)$, so Lemma 11.3 
implies $0<\angle\left(f^{\circ n}(e), f^{\circ n}\left(e^{\prime}\right)\right) \leq 1 / d\left(f^{\circ n}(\omega)\right)$ for all $n$. This is clearly impossible as we have expansiveness in the Böttcher coordinate.

The only possibility left is that for some $n$ there is a folding critical Fatou point in $\left[f^{\circ n}\left(v_{\mathcal{A}}\right), f^{\circ n}\left(v_{\mathcal{A}^{\prime}}\right)\right]$. Without loss of generality we take $n=0$. Again there are two edges $e, e^{\prime}$ near $\omega$ pointing to $v_{\mathcal{A}}, v_{\mathcal{A}}^{\prime}$. The angle between these edges is a multiple of $1 / d(\omega)$, so bearing in mind that $\mathcal{F}_{\omega}$ is constructed by taking the other ends of the preferred $e_{0}, e_{1}, \ldots, e_{d(\omega)-1}$, a simple application of Lemma 9.3 tells us that one of the edges (and hence both) is to be picked among $e_{0}, e_{1}, \ldots, e_{d(\omega)-1}$, the preferred ones, just to keep alive the possibility that the arguments share right address. In that event Lemma 10.2 implies that necessarily one of them, say $\mathcal{A}$, must be supporting for $\omega$ while the other is not. But $\mathcal{A}$ is periodic, and inside $\mathcal{F}_{\omega}$ only $\mathcal{D}\left(\omega, e_{0}\right)$ is; hence we get $\mathcal{A}=\mathcal{D}\left(\omega, e_{0}\right)$. As a by-product we conclude that $\omega$ and $v_{\mathcal{A}}$ share period. Angle considerations then imply $e^{\prime}=e_{1}$. Let $\hat{\mathcal{A}}=\mathcal{D}\left(\omega, e_{1}\right)$ be based at $\hat{v}$, the other endpoint of $e_{1}$. Then by definition we have $f(\hat{v})=f\left(v_{\mathcal{A}}\right)$. Let $m$ be a high common multiple of the periods of $\mathcal{A}$ and $\mathcal{A}^{\prime}$ (and a fortiori of $\omega$ ). Then $f^{\circ m}(\hat{v})=f^{\circ m}\left(v_{\mathcal{A}}\right)=v_{\mathcal{A}}$ does not belong to $\left[f^{\circ m}(\omega), f^{\circ m}\left(v_{\mathcal{A}^{\prime}}\right)\right]=$ $\left[\omega, v_{\mathcal{A}^{\prime}}\right]$. Nevertheless $\hat{v}$ is contained in $\left[\omega, v_{\mathcal{A}^{\prime}}\right]$. We deduce that there is a last $k \in\{0, \ldots, m-1\}$ for which $f^{\circ k}(\hat{v})$ is in $\left[f^{\circ k}(\omega), f^{\circ k}\left(v_{\mathcal{A}^{\prime}}\right)\right]$. According to Lemma 5.2, this can only happen when the last segment contains a folding critical point. Let $\sigma$ be the first folding critical point in the oriented segment from $f^{\circ k}(\omega)$ to $f^{\circ k}\left(v_{\mathcal{A}^{\prime}}\right)$. Key here is to recognize that $\sigma$ stands between $f^{\circ k}\left(v_{\mathcal{A}}\right)$ and $f^{\circ k}\left(v_{\mathcal{A}^{\prime}}\right)$, and as such is of Fatou type (else we are done thanks to Lemma 11.2). Another important detail is that $f^{\circ k}(\hat{\mathcal{A}})$ cannot support the Fatou critical point $\sigma$ since it already supports $f^{\circ k}(\omega)$. From this choice we conclude readily that $f(\sigma)$ stands in the same connected component of $H-\left\{f^{\circ k+1}(\omega)\right\}$ as $f^{\circ k+1}(\hat{v})$, a component where by definition of $k$ we should be unable to place $f^{\circ k+1}\left(v_{\mathcal{A}^{\prime}}\right)$. In particular, the distance between $f^{\circ k+1}\left(v_{\mathcal{A}^{\prime}}\right)$ and $f(\sigma)$ is greater than 1 . This implies that $f^{\circ k+1}\left(\mathcal{A}^{\prime}\right)$ is not a supporting access for $f(\sigma)$. This renders impossible for $f^{\circ k}\left(\mathcal{A}^{\prime}\right)$ to support $\sigma$. But this is déjà vu. We have a Fatou critical point $\sigma$ sitting between $f^{\circ k}\left(v_{\mathcal{A}}\right)$ and $f^{\circ k}\left(v_{\mathcal{A}^{\prime}}\right)$ and such that neither $f^{\circ k}(\mathcal{A})$ nor $f^{\circ k}\left(\mathcal{A}^{\prime}\right)$ supports $\sigma$. A reasoning similar to one given in the last paragraph shows that $f^{\circ k}(\mathcal{A})$ and $f^{\circ k}\left(\mathcal{A}^{\prime}\right)$ belong to different connected components of $\mathbb{T}-\mathcal{F}_{\sigma}$.

COROLlary 11.5. The critical portrait $\Theta=(\mathcal{F}, \mathcal{J})$ satisfies condition (c6).

Proof. Let a periodic $\lambda$ satisfy $s_{\Theta}^{+}(\lambda)=s_{\Theta}^{+}(\mathcal{A})$, where $\mathcal{A}$ is a marked access. Using Proposition 9.9 we can assume that the access associated to $\lambda$ is present in the tree. By Lemma 11.4 this access is supported at $v_{\mathcal{A}}$. According to Lemma 10.2 it can only be $\mathcal{A}$. 
REMARK 11.6. The proof of Corollary 11.5 is mischievously simple. We are employing Proposition 9.9 to assume that the access associated to $\lambda$ is already present in the tree. As was hinted before, this seemingly natural fact is not trivial at all.

To work out (c7) we must first study when two accesses are based at the same Julia point. We start with a simple criterion.

LEMma 11.7. Two accesses have the same left address if and only if they are supported at the same subtree of the preferred $T^{*}$.

Proof. Everything was defined in order for this to be true. (Compare also Lemmas 10.2 and 10.3.)

Lemma 11.8. Let $\mathcal{A}, \mathcal{A}^{\prime}$ be accesses. If $s_{\Theta}^{-}(\mathcal{A})=s_{\Theta}^{-}\left(\mathcal{A}^{\prime}\right)$, then $v_{\mathcal{A}}=v_{\mathcal{A}^{\prime}}$.

Proof. According to the last lemma, there is always a member of $T^{*}$ where we can find simultaneously $f^{\circ n}\left(v_{\mathcal{A}}\right)$ and $f^{\circ n}\left(v_{\mathcal{A}}^{\prime}\right)$. The result follows from Proposition 6.5.

Corollary 11.9. Two periodic accesses $\mathcal{A}, \mathcal{A}^{\prime}$ are based at the same point if and only if $s_{\Theta}^{-}(\mathcal{A})=s_{\Theta}^{-}\left(\mathcal{A}^{\prime}\right)$.

Proof. Since the accesses are periodic, so are their base points. Thus, they are regular Julia points and as such each belongs to barely one subtree in $T^{*}$. Suppose first the points are the same. Lemma 11.7 tells us that they share a unique left address. The same is true for their forward iterates, and the corresponding left symbol sequences agree. The converse is Lemma 11.8.

Lemma 11.10. Let $\mathcal{A}$ be an access based at a Julia critical point $c$. Then there is a marked access $\mathcal{A}_{c} \in \mathcal{J}_{c}$ such that $a_{\Theta}^{-}(\mathcal{A})=a_{\Theta}^{-}\left(\mathcal{A}_{c}\right)$.

Proof. In fact, the point $c$ belongs to exactly $d(c)$ members of $T^{*}$. As the $d(c)$ elements of $\mathcal{J}_{c}$ have different left addresses, by Lemma 11.7 they are supported in different subtrees. But $\mathcal{A}$ is based at $c$, so it must be supported in one of these $d(c)$ subtrees as well.

Lemma 11.11. Suppose $a_{\Theta}^{-}(\mathcal{A})=a_{\Theta}^{-}\left(\mathcal{A}^{\prime}\right)$. Then $v_{\mathcal{A}}=v_{\mathcal{A}^{\prime}}$ if and only if $v_{f(\mathcal{A})}=v_{f\left(\mathcal{A}^{\prime}\right)}$.

Proof. Once we write $v_{f(\mathcal{A})}=f\left(v_{\mathcal{A}}\right)=f\left(v_{\mathcal{A}^{\prime}}\right)=v_{f\left(\mathcal{A}^{\prime}\right)}$, one direction is obvious. The other is true thanks to Lemmas 11.7 and 6.2.

Instead of proving condition (c7) directly, we will take a small detour and relate what we have accomplished with the $\sim_{l}$ relation (cf. Definition 14.9).

Lemma 11.12. Suppose we have $a_{\Theta}^{-}(\mathcal{A})=a_{\Theta}^{-}\left(\mathcal{A}^{\prime}\right)$. Then $\mathcal{A} \sim_{l} \mathcal{A}^{\prime}$ if and only if $f(\mathcal{A}) \sim_{l} f\left(\mathcal{A}^{\prime}\right)$. 
Proof. This property is combinatorial and has nothing to do with dynamics. We are just copying Lemma 14.10 for the benefit of the readers.

Proposition 11.13. Given accesses $\mathcal{A}, \mathcal{A}^{\prime}$, we have $v_{\mathcal{A}}=v_{\mathcal{A}^{\prime}}$ if and only if $\mathcal{A} \sim_{l} \mathcal{A}^{\prime}$.

Proof. First suppose $\mathcal{A} \sim_{l} \mathcal{A}^{\prime}$. We will prove that if $s_{\Theta}^{-}(\mathcal{A}), s_{\Theta}^{-}\left(\mathcal{A}^{\prime}\right)$ are related by either (11) or (12) in Definition 14.9, then $v_{\mathcal{A}}$ and $v_{\mathcal{A}^{\prime}}$ are the same. If $s_{\Theta}^{-}(\mathcal{A})=s_{\Theta}^{-}\left(\mathcal{A}^{\prime}\right)$, this is a consequence of Corollary 11.9 and Lemma 11.11. When (12) is satisfied, that is, if $s_{\Theta}^{-}(\mathcal{A}), s_{\Theta}^{-}\left(\mathcal{A}^{\prime}\right)$ agree up to the $j$ th position and $f^{\circ j}(\mathcal{A})$ and $f^{\circ j}\left(\mathcal{A}^{\prime}\right)$ are both in $\mathcal{J}_{c}$, then we can apply Lemma 11.11 $j$ times knowing that $f^{\circ j}(\mathcal{A}), f^{\circ j}\left(\mathcal{A}^{\prime}\right)$ are based at the same critical point.

The reverse implication is settled by induction on the number of steps required by $v_{\mathcal{A}}$ to become periodic. If $v_{\mathcal{A}}$ is periodic (this is the inductive seed), then we have $s_{\Theta}^{-}(\mathcal{A})=s_{\Theta}^{-}\left(\mathcal{A}^{\prime}\right)$ due to Corollary 11.9. Therefore 11 is satisfied. Now suppose $f(\mathcal{A}) \sim_{l} f\left(\mathcal{A}^{\prime}\right)$ (this is the inductive hypothesis). If $a_{\Theta}^{-}(\mathcal{A})=a_{\Theta}^{-}\left(\mathcal{A}^{\prime}\right)$, then we apply Lemma 11.12 and conclude $\mathcal{A} \sim_{l} \mathcal{A}^{\prime}$. Otherwise $c=v_{\mathcal{A}}$ is critical and Lemma 11.10 provides accesses $\mathcal{C}, \mathcal{C}^{\prime} \in \mathcal{J}_{c}$ subject to $a_{\Theta}^{-}(\mathcal{A})=a_{\Theta}^{-}(\mathcal{C})$ and $a_{\Theta}^{-}\left(\mathcal{A}^{\prime}\right)=a_{\Theta}^{-}\left(\mathcal{C}^{\prime}\right)$. However, the inductive hypothesis together with Lemma 11.12 implies $\mathcal{A} \sim_{l} \mathcal{C}$ and $\mathcal{A}^{\prime} \sim_{l} \mathcal{C}^{\prime}$. As by definition we have $\mathcal{C} \sim_{l} \mathcal{C}^{\prime}$, the result follows from transitivity.

Corollary 11.14. The critical portrait $\Theta=(\mathcal{F}, \mathcal{J})$ satisfies condition (c7).

Proof. Let $\theta=\mathcal{A} \in \mathcal{J}_{l}$ and $\theta^{\prime}=\mathcal{A}^{\prime} \in \mathcal{J}_{k}$. If $s_{\Theta}^{-}\left(d^{i} \theta\right)=s_{\Theta}^{-}\left(\theta^{\prime}\right)$, then $f^{\circ i}(\mathcal{A}) \sim_{l} \mathcal{A}^{\prime} \in \mathcal{J}_{k}$ due to the last proposition. If $i=0$, then (clearly) $\mathcal{J}_{l}=\mathcal{J}_{k}$. Else, owing to the hierarchic selection, we have $f^{\circ i}(\mathcal{A}) \in \mathcal{J}_{k}$. Finally, as different marked accesses from $\mathcal{J}_{k}$ yield different left addresses, we get $f^{\circ i}(\mathcal{A})=\mathcal{A}^{\prime}$.

Proposition 11.15. The formal critical portrait $\Theta=(\mathcal{F}, \mathcal{J})$ constructed in the last section is admissible.

Proof. This follows from Corollaries 11.5 and 11.14.

12. Back to Hubbard trees. The critical portrait associated to an abstract Hubbard tree is admissible. Therefore, according to Theorem 14.6, there is a unique postcritically finite polynomial, from now on $f$, that materializes this portrait.

Thus, we have two Hubbard trees (we might think as well of two families of extensions): the original abstract tree $H$, and the polynomial related $H_{f}$. If we manage to prove that their structures are the same, then Theorem 1.1 is true.

We must show that accesses at Julia points are in natural correspondence between trees. That is, a group of rational rays landing together should be 
identified with all the accesses based at a Julia point. On the other hand, because the construction around Fatou vertices is overdetermined (yet still coherent), there is not much problem to compare the layouts next to Fatou points either.

Lemma 12.1. Given rational angles $\theta, \theta^{\prime}$, take an abstract extension of $H$ in which the corresponding accesses are present. These accesses share the same base point in $H$ if and only if the rays $R_{\theta}$ and $R_{\theta^{\prime}}$ land at the same point of $J(f)$.

Proof. This result is trivial once the accesses corresponding to these angles are present: to share a base point in the lattice of Hubbard trees and to land at the same point in $J(f)$ are identical questions in the $\sim_{l}$ language.

Given a rational argument $\theta$, we write $p_{\theta}$ for the landing point of $R_{\theta}$, and $v_{\theta}$ for the vertex that supports the access (if it exists) in $H$. Lemma 12.1 tells us that $p_{\theta}$ and $v_{\theta}$ correspond perfectly. More is true. As the accesses about the base points are equal, the same extends to the branches in between. (They might be physically present, or they might be "virtual". For the time being it does not matter; we will explore this more deeply in Section 13.)

Lemma 12.2. Let $\theta$ be a periodic argument. Suppose that the landing point $p_{\theta}$ of $R_{\theta}$ (respectively, the base point $v_{\theta}$ of the access associated with $\theta$ ) is a vertex in $H_{f}$ (respectively, in $H$ ). Then the arguments of the rays that land at $p_{\theta}$ are precisely the arguments of the accesses based at $v_{\theta}$.

Proof. Extend the abstract tree $H$ to include each and every access associated with an argument of the rays landing at $p_{\theta}$ and apply Lemma 12.1.

Of course, by looking at the inverses we deduce the same for any rational arguments. We can also work with branches.

Lemma 12.3. Take $\theta$ and $\tau$ rational and not $\sim_{l}$ related. Assume that $v_{\theta}$ and $v_{\tau}$ belong to $H$. Then the branch that stems from $v_{\theta}$ and contains $v_{\tau}$ is the same as the one that stems from $p_{\theta}$ and contains $p_{\tau}\left(\right.$ in $\left.H_{f}\right)$.

Proof. Cyclically arrange as $\theta_{0}=\theta<\theta_{1}<\cdots<\theta_{n-1}<\theta_{n}=\theta+1$ all the arguments $\sim_{l}$-related to $\theta$. As $\tau$ is not listed, we have $\theta_{i}<\tau<\theta_{i+1}$ for some $i$. In both tree models the vertex associated to $\tau$ is trapped between the accesses labeled $\theta_{i}$ and $\theta_{i+1}$.

Fatou vertices can also be set into one-to-one correspondence (for instance, make use of supporting accesses to relate them). In both models we have distinguished internal directions that define Böttcher coordinates. By definition the zero internal directions correspond for free. For all others we have the following. 
Lemma 12.4. Let $\omega$ be a Fatou vertex. Take $\theta$ to be rational. The Böttcher argument of the branch that stems from $\omega$ and contains $v_{\theta}$ (respectively $\left.p_{\theta}\right)$ is the same in $H$ and in $H_{f}$.

Proof. Let us look first at the particular case where $e=\left[\omega, p_{\theta}\right]$ is an internal ray that eventually catches up with a preferred internal direction of a Fatou critical point. For example if $e$ is the zero ray near the critical point $\omega$, then by definition $p_{\theta}$ is the base point of the access $\lambda=\mathcal{D}(e, \omega)$, and we must have $p_{\theta}=p_{\lambda}$. Now, supporting arguments are by construction the same in the abstract and the concrete models. Therefore $v_{\lambda}$ is contiguous to $\omega$ in the abstract tree through the zero internal ray in the Böttcher coordinate. We now invoke Lemma 12.1 to find out that $v_{\theta}$ is actually $v_{\lambda}$, and we are done. The case where $f^{\circ n}(e)$ is the zero ray can then be worked out by induction.

Else, with the help of the particular cases worked out above and Lemma 12.3 we trap angles. This means that the relative positions of the $\operatorname{arcs}\left[\omega, p_{\theta}\right]$ and $\left[\omega, v_{\theta}\right]$ with respect to the edges incident at $\omega$ are the same, for example in inverse trees. According to Theorem 14.4 this relative position is enough to assign the same coordinate once we prescribe the zero direction.

Proof of Theorem 1.1. In this section we have just proved that the Hubbard tree associated to $f$ is the same as the starting one. The unique realization of critical portraits implies the same for Hubbard trees.

13. Loose ends. The successful conclusion of our work hinges now only on Proposition 9.9. Along this section we fix a homogeneous Hubbard tree with at least one Julia vertex. We assume without loss of generality that the tree is fit and that no Fatou vertex is an end. For practical purposes we take for granted that at a Fatou vertex of degree $n$ there are $n$ segments that collapse under $f$. This last property can be achieved already in the first inverse tree.

We start with a technical lemma to narrow down our search for an access associated to a periodic argument. Remember that outside the minimal tree all periodic points must be ends (cf. Proposition 5.11).

Lemma 13.1. Let $m \geq 1$ be an integer. The number of periodic vertices of period $m$ in any canonical extension is bounded by a constant that depends on the minimal tree and on $m$.

Proof. All periodic points of period $m$ are already vertices in the minimal tree or have incidence one or two. The vertices in the minimal tree are accounted for. At vertices not in the minimal tree (or not marked) the accesses have period $m$ or $2 m$. However, once we assign coordinates, the dynamics of $\theta \mapsto d \theta$ allows only a finite number of periodic orbits of period $m$ or $2 m$. 
In particular, we can always detect in the underlying topological tree where periodic points of period $m$ or less can be canonically placed. There are several ways to find those locations; for instance, Markov partition techniques are good enough.

EXAMPLE 13.2. As an example, starting from the minimal tree of the rabbit we construct extensions where we can observe specific orbits. First we must comply with the basic framework stated at the beginning of this section: all ends should be of Julia type and there must be a pair of segments that fold under iteration near the unique critical point. This is easy to achieve (as a subtree in the third inverse) and is shown in Figure 13.1, left. For the benefit of our readers we have indicated the arguments of the rays that land at such points.
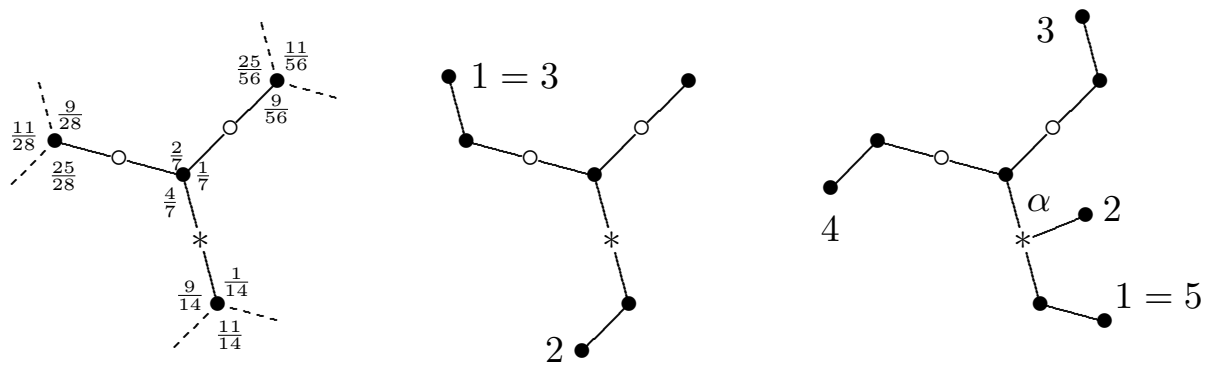

Fig. 13.1. A rabbit with no Fatou ends and a couple of extensions

Let us see where we can place the period two orbit $1 / 3 \mapsto 2 / 3 \mapsto 1 / 3$. Keeping in mind the arguments in the figure, we trap $1 / 3$ between $9 / 28$ and $11 / 28$, and $2 / 3$ between $9 / 14$ and $11 / 14$. As $v_{1 / 3}$ is obviously not in the minimal tree, a branch at $v_{9 / 28}$ must point to it. In which direction? Numbers tell. The same with $v_{2 / 3}$. Still, we have not proved anything yet. The dynamical tree in Figure 13.1, center, (with the pair of period two points appended) is expanding, so we are facing a Hubbard tree. The accesses also have period two, and therefore they can only be associated with the angles $1 / 3$ and $2 / 3$.

Now we work out a more complex case: we append $v_{1 / 15}, v_{2 / 15}, v_{4 / 15}, v_{8 / 15}$ so that they form the period four Julia type orbit associated with the labels. First we start trapping values as

$$
\frac{11}{14}<\frac{1}{15}<\frac{1}{14}, \quad \frac{1}{14}<\frac{2}{15}<\frac{1}{7}, \quad \frac{11}{56}<\frac{4}{15}<\frac{25}{56}, \quad \frac{11}{28}<\frac{8}{15}<\frac{25}{28} .
$$

From these relations we immediately guess where $v_{1 / 15}, v_{4 / 15}, v_{8 / 15}$ ought to stand. However, there is a small inconvenience with $v_{2 / 15}$ since the trapping values $1 / 14,1 / 7$ are not supported at the same Julia point. This implies that somewhere in the path $\left[v_{1 / 14}, v_{1 / 7}\right]$ a new road opens. This is only 
possible at the Fatou critical point, where a new edge makes an angle (still unknown) of $\alpha \in(0,180)$ with respect to the reference direction (compare Figure 13.1, right). To turn the obvious dynamics into a Hubbard tree we push forward the dynamics and solve the equation $2 \alpha=180$. Why can we ensure that the new orbit must be labeled with the angles in waiting? The answer is somehow trivial and can be given without further calculation. In what multiplication by 2 in the unit circle concerns, the only period four argument between $1 / 14$ and $1 / 7$ is $2 / 15$. This implies that $v_{2 / 15}$ is where it should be, and, of course, the other vertices too.

Proof of Proposition 9.9. To alleviate matters, suppose all points of period $m$ potentially belonging to the minimal tree are detected and marked as vertices. We arrange cyclically the arguments present in $H$ as $\alpha_{0}, \alpha_{1}, \ldots, \alpha_{k}$ $=\alpha_{0}$. Now let $\theta \mapsto d \theta \mapsto \cdots \mapsto d^{m} \theta=\theta$ be the period $m$ orbit that we want to append (if not already present, else we are done). Let $M$ be a number greater than $m$ and the periods of all periodic accesses in $H$. As the preimages of $\alpha_{0}$ under multiplication by $d$ are dense in $\mathbb{T}$, by taking lots of inverses of $H$ we assume that each closed interval $I_{i}=\left[\alpha_{i}, \alpha_{i+1}\right]$ measures $1 / d^{2 M}$ or less. This is important as each $I_{i}$ contains at most one periodic element of period $M$ or less thanks to size considerations. In particular, it intersects at most once the orbit of $\theta$. This is helpful: if we have $\theta \in I_{j}$, for some $j$, and if we manage to construct an extension with a periodic element of period $m$ or less associated to some angle contained in $I_{j}$, then without further ado the argument is exactly $\theta$, and we are done.

Next we decorate $H$. We plan to take several inverses of $H$ and then restrict back to $H$. We are interested in the potential branching at Julia points. Formally this is done as follows. There is a suitable iterated inverse $\operatorname{inv}^{o l}(H)$ in which the incidence at all $v \in V_{H}$ is saturated. We return to $H$ and in each $v \in V_{H}$ we graft a sprout following the reported pattern. This renders a configuration of virtual directions followed by virtual accesses. By construction, virtual accesses also have a right to a faithful argument.

As remarked above, every $d^{k} \theta$ in the orbit of $\theta$ can be placed inside an interval $\left(\alpha_{i_{k}}, \alpha_{i_{k}+1}\right)$ with nonperiodic extremes. Let $v_{k}$ be the vertex where $\alpha_{i_{k}}$ is supported and $v_{k}^{\prime}$ where $\alpha_{i_{k}+1}$ is. Neither of them is periodic. There are three cases to be considered: Case 1 when these two vertices are the same, Case 2 there is an edge with endpoints $v, v^{\prime}$, and, finally, Case 3 when a Fatou vertex interposes between them. (This exhausts all possibilities due to fitness.)

Now we show how to reduce the second possibility to either the first or the third. Consider the preimages of $\alpha_{i_{k}}, \alpha_{i_{k}+1}$ under multiplication by $d^{m}$ given by $\beta=d^{k} \theta-\left(d^{k} \theta-\alpha_{i_{k}}\right) / d^{m}, \beta^{\prime}=d^{k} \theta+\left(\alpha_{i_{k}+1}-d^{k} \theta\right) / d^{m}$, respectively 

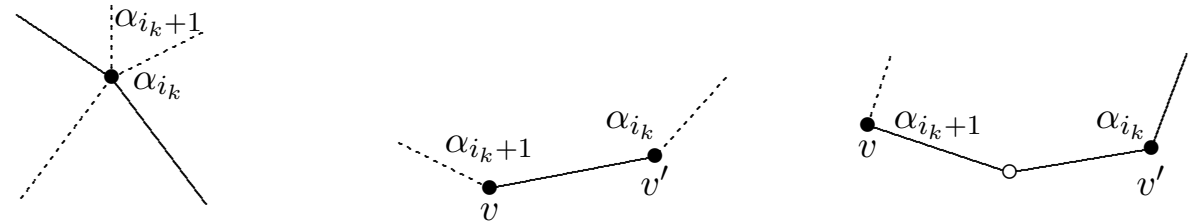

Fig. 13.2. The three configurations

(remember $\theta$ has period $m$ ). Those values are subject to

$$
\alpha_{i_{k}}<\beta<d^{k} \theta<\beta^{\prime}<\alpha_{i_{k}+1},
$$

as is easy to check. In the tree $\operatorname{inv}^{\circ m}(H)$ there are accesses reserved for $\beta, \beta^{\prime}$. If the interval $\left[v_{\beta}, v_{\beta^{\prime}}\right]$ were physically contained in $H$, then it must be properly included in $\left[v_{\alpha_{i_{k}}}, v_{\alpha_{i_{k}+1}}\right]$. Therefore (as $v_{\beta}$ maps to $v_{\alpha_{i_{k}}}$ and $v_{\beta}$, to $v_{\alpha_{i_{k}}}$ under $f^{\circ m}$ ) there exists a period $m$ point inside $\left[v_{\alpha_{i_{k}}}, v_{\alpha_{i_{k}+1}}\right] \subset H$, contrary to our assumption. This proves that at least one among $v_{\beta}, v_{\beta^{\prime}}$ can only be materialized in a potential inverse. We draw an arc between the said point and $v_{\alpha_{i_{k}}}$ and denote by $\tilde{v}$ the first point that tops $H$. Include $\tilde{v}$ and its orbit in $H$. Make this vertex fit when it is of Fatou type and graft sprouts when it is a Julia point. Anyway, as we have not appended new periodic points or interfered with Case 1 or Case 3 along the orbit of the preperiodic point $\tilde{v}$, we see that Case 2 no longer holds and we are reduced either to Case 1 or 3 .

In Case 1 there is a virtual direction between $\alpha_{i_{k}}$ and $\alpha_{i_{k}+1}$. Append an edge there. Call the new endpoint $v_{d^{k} \theta}$. The angles are copied from the virtual distribution. In Case 3 we do the same but this time near the Fatou vertex. However, no internal argument is assigned at the time. For the sake of discussion, in both cases we denote by $\tilde{v}_{d^{k} \theta}$ the vertex that connects $v_{d^{k} \theta}$, the new vertex, to the old tree.

When we map $v_{d^{k} \theta}$ to $v_{d^{k+1} \theta}$, we are almost done but for a small detail: the angles at Fatou vertices $\tilde{v}_{d^{k} \theta}$ are yet to be defined. If $f\left(\tilde{v}_{d^{k} \theta}\right) \neq \tilde{v}_{d^{k+1} \theta}$, there is no problem: the image of $\left[\tilde{v}_{d^{k} \theta}, v_{d^{k} \theta}\right]$ (which is $\left[f\left(\tilde{v}_{d^{k} \theta}\right), v_{d^{k+1} \theta}\right]$ and includes $\left.\left[f\left(\tilde{v}_{d^{k} \theta}\right), \tilde{v}_{d^{k+1} \theta}\right]\right)$ branches from $f\left(\tilde{v}_{d^{k} \theta}\right)$ at a well defined internal angle; we simply copy this value. These angles can be inductively pulled back to reach every Fatou point in the orbit unless they itself configure a loop. However, this is only possible if the Fatou vertices are periodic. But if this is the case, then the edges $\left[\tilde{v}_{d^{k} \theta}, v_{d^{k} \theta}\right]$ are periodic while their immediate neighbors - around $\tilde{v}_{d^{k} \theta}$-are not. Therefore, the first return maps are finite expanding maps. In light of Theorem 14.4, they can be angled and we recover the Böttcher coordinates. From that we read off the missing data.

The extension is canonical because for any extension including the vertex $v_{d^{k} \theta}$, this point must be found in a branch that stems from $\tilde{v}_{d^{k} \theta}$ at a well known direction. 
This completes the proof of Proposition 9.9, and our work can be called to an end. Nevertheless, we still have time for a last result of interest.

COROLlary 13.3. Every expanding extension of an abstract Hubbard tree is canonical.

Proof. Given any extension, we assign to every periodic access an argument (compare Proposition 9.7). On the other hand, to the minimal tree we can append the same periodic orbits using Proposition 9.9. Finally, we take a finite number of inverses and restrict if necessary to recognize that the process is canonical.

14. Appendix. Finite cyclic expanding maps. If we wish, we can always think of a selfmap of a finite cyclic set as multiplication by some integer $d$ in the unit circle. The problem is that $d$ is far from what we want to call the degree of our map since it is normally taken huge to simplify matters. In this appendix we define a concept of degree suitable for our purposes and just then state conditions to guarantee that our data can be "angled".

Given $k \geq 1$ and $d>1$, consider a cyclic set $X$ with $k d$ elements. The idea is to define as economically as possible the concept of orientation preserving degree $d$ selfmap onto a proper subset of $X$. Take any $a \in X$ and rename the elements as $a_{0}, a_{1}, \ldots, a_{k d-1}$ starting with $a_{0}=a$. An orientation preserving degree $d$ selfmap of $X$ is a function that satisfies $f\left(a_{i}\right)=f\left(a_{j}\right)$ if and only if $i \equiv j(\bmod k)$, and $f\left(a_{i+1}\right)$ follows $f\left(a_{i}\right)$ in the induced order in $f(X)=\left\{f\left(a_{0}\right), \ldots, f\left(a_{k-1}\right)\right\}$. Note that for simplicity any element in the image has exactly $d$ preimages. The concept is independent of the starting $a$.

Not all orientation preserving degree $d$ selfmaps of a finite cyclic set can be thought of as multiplication by $d$ restricted to an invariant subset of $\mathbb{T}$. As a matter of fact, multiplication by $d>1$ is locally expanding, and this condition must be captured somehow. We say that $f$, as above, is expanding if the image of successive elements eventually cease to be consecutive. In symbols, given $p_{0} \in X$ with successor $p_{1} \in X$, there is an $n \geq 1$ such that $f^{\circ n}\left(p_{1}\right)$ does not follow $f^{\circ n}\left(p_{0}\right)$ in $X$. Even if there are several subsets that allow cyclic orders, the expanding condition is to be tested in $X$, the original set. As usual it is enough to work with adjacent periodic elements.

In this context, different elements cannot stay dynamically close forever. If the distance between consecutive members increases, then other elements are also pushed apart. This can only stop when the gap reaches an adequate threshold where injectivity no longer holds. All this is made precise next.

The domain of $f$ can be subdivided into $d$ clusters of size $k$ starting anywhere. Given $b \in X$ we again write $b_{0}=b, \ldots, b_{d k-1}$ cyclically. For any $a$ we must have $a=b_{m}$ for some $m$, and so also $a=b_{i k+j}$ where $0 \leq i<d$ 
and $0 \leq j<k$. Set $Q(b, a)=i$ (this $Q$ is a sort of arithmetic quotient).

Lemma 14.1. Let $f: X \rightarrow X$ be expanding. Then $Q\left(f^{\circ n}(a), f^{\circ n}(b)\right)=0$ for all $n \geq 0$ if and only if $a=b$.

Proof. If $a=b$, then $f^{\circ n}(a)=f^{\circ n}(b)$, and $Q\left(f^{\circ n}(a), f^{\circ n}(b)\right)=0$ by definition.

If we suppose $a \neq b$ and $Q(a, b)=0$, then the directed distance between $f^{\circ n}(a)$ and $f^{\circ n}(b)$ eventually grows until it is at least $k$. At that point the corresponding $Q\left(f^{\circ n}(a), f^{\circ n}(b)\right)$ does not vanish.

Corollary 14.2. Let $a \neq b$. If $Q\left(f^{\circ n}(a), f^{\circ n}(b)\right)=d-1$ for all $n$, then $f$ is not expanding.

Proof. Note that $Q(p, q)=d-1$ implies $Q(q, p)=0$ unless $f(p)=$ $f(q)$. After this remark, it is clear that $Q\left(f^{\circ n}(a), f^{\circ n}(b)\right)=d-1$ for all $n$ implies $Q\left(f^{\circ n}(b), f^{\circ n}(a)\right)=0$ for all $n$. Due to the last lemma, $f$ cannot be expanding.

We say that $f$ can be angled if there exists an order preserving embedding $\phi: X \rightarrow \mathbb{T}$ for which $d \phi(p) \equiv \phi(f(p))(\bmod 1)$. In that case we define $\angle(a, b)=\phi(b)-\phi(a)$. In this context $Q(a, b)$ can be interpreted as the first digit in the base $d$ expansion of the chief representative of $\angle(a, b)$.

An angled function is obviously expanding. Before we turn to the converse, let us work out an example.

Example 14.3. Consider the dynamical system hinted by the data below. We will show how to find coordinates so that this map becomes multiplication by 3 .

$$
\begin{aligned}
& A, A^{\prime}, A^{\prime \prime} \mapsto B \\
& B, B^{\prime}, B^{\prime \prime} \mapsto C \\
& C, C^{\prime}, C^{\prime \prime} \mapsto D \\
& D, D^{\prime}, D^{\prime \prime} \mapsto A
\end{aligned}
$$

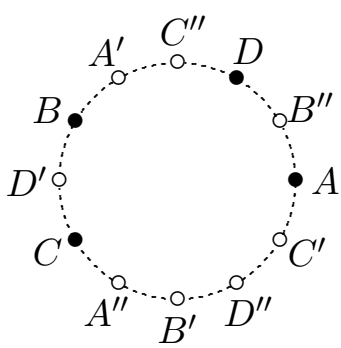

First we try to get a veritable angle function. The angle $\angle\left(A, A^{\prime}\right)$ from $A$ to $A^{\prime}$ should be $1 / 3$, equal also to $\angle\left(A^{\prime}, A^{\prime \prime}\right)$ and $\angle\left(A^{\prime \prime}, A\right)$. The same holds for all other labels. To find for example $\angle\left(A, B^{\prime}\right)$ we rely on partial estimates. By inspection $\angle\left(A, B^{\prime}\right)$ is trapped between $2 / 3$ and 1 . Next we push forward in order to determine the base 3 expansion of $\angle\left(A, B^{\prime}\right)$. To achieve our goal we write both orbits in parallel as

$$
\begin{gathered}
A \mapsto B \mapsto C \mapsto D \mapsto A \mapsto B \mapsto C \mapsto D \mapsto A, \\
B^{\prime} \mapsto C \mapsto D \mapsto A \mapsto B \mapsto C \mapsto D \mapsto A \mapsto B .
\end{gathered}
$$


Thus, the successive digits in base 3 are $2,0,1,2,1,0,1,2,1$. From there on the numbers repeat in packs of four taking 0121 as pattern. As four "decimal" places represent $1 / 3^{4}=1 / 81$, the word 0121 is equivalent to $16=0.27+1.9+2.3+1$. Hence, we get

$$
\angle\left(A, B^{\prime}\right)=\frac{2}{3}+\frac{1}{3}\left(\frac{16}{3^{4}}+\frac{16}{3^{8}}+\cdots\right)=\frac{2}{3}+\frac{1}{3} \frac{16}{3^{4}-1}=\frac{11}{15} .
$$

As an application, we find a faithful coordinate $\theta_{A}$ for $A$. Our work above immediately implies $\angle(A, B)=11 / 15-1 / 3=2 / 5$. Then, as $\angle(A, B)=$ $\angle(A, f(A))$ can only be $3 \theta_{A}-\theta_{A}$, we assign to $A$ the argument $1 / 5$. For any other $P$ we define $\theta_{P}=\theta_{A}+\angle(A, P)$.

Theorem 14.4. A degree $d>1$ orientation preserving $f: X \rightarrow X$ can be angled if and only if it is expanding. If so, there are precisely $d-1$ different ways to assign coordinates.

Proof. Given $a, b \in X$, we dynamically find the size of the gap between these two elements in base $d$ : define the $n$th entry of $\angle(a, b)$ as $Q\left(f^{\circ n}(a), f^{\circ n}(b)\right)$. We get $\angle(a, b)=0$ if and only if $Q\left(f^{\circ n}(a), f^{\circ n}(b)\right)=0$ for all $n$. Under the expanding condition this can only be true provided $a=b$. On the other hand, $\angle(a, b)=1$ implies $Q\left(f^{\circ n}(a), f^{\circ n}(b)\right)=d-1$ for all $n$, which is impossible in light of Corollary 14.2.

Up to this point we have a well defined oriented angle for every pair of elements. To materialize coordinates, we fix $a \in X$ and measure the angle between $a$ and $f(a)$ as $\angle(a, f(a))$. If $\theta_{a}$ corresponds to $a$, then $d \theta_{a}$ is related to $f(a)$. The equation

$$
d \theta_{a}-\theta_{a}=\angle(a, f(a)),
$$

for unknown $\theta_{a}$, can be solved in exactly $d-1$ ways. To get the remaining values it is enough to comply with $\theta_{b}=\theta_{a}+\angle(a, b)$.

Critical portraits. We recall the ideas behind the theory of critical portraits for postcritically finite polynomials following [P3] closely. For convenience, we reproduce the definitions and statements with proofs omitted. Here $f: \mathbb{C} \rightarrow \mathbb{C}$ represents always a postcritically finite polynomial of degree $d \geq 2$.

From a bounded Fatou component $U$ take a boundary point $p \in \partial U$. When we order counterclockwise the external rays $R_{\theta_{1}}, \ldots, R_{\theta_{k}}$ landing at $p$, the complex plane splits into $k$ pieces. Whenever $U$ fits inside the region determined by $R_{\theta_{1}}$ and $R_{\theta_{2}}\left(\theta_{2}=\theta_{1}\right.$ in case a single ray lands at $\left.p\right)$, the argument $\theta_{1}$ becomes by definition the (left) supporting argument of the Fatou component $U$ at $p$, and $R_{\theta_{1}}$ the supporting ray.

Given a postcritically finite degree $d \geq 2$ polynomial $f$, we assign to every critical point a finite subset of $\mathbb{Q} / \mathbb{Z}$ and construct a critically marked 
polynomial $(f, \Theta)$, where the marking $\Theta=(\mathcal{F}, \mathcal{J})$ is actually a pair of families of subsets of the circle. Here $\mathcal{F}=\left\{\mathcal{F}_{1}, \ldots, \mathcal{F}_{l}\right\}$ is a finite family of subsets where each $\mathcal{F}_{k}$ stands for a finite set of arguments associated with the critical point $\omega_{k}$ in the Fatou set, whereas $\mathcal{J}=\left\{\mathcal{J}_{1}, \ldots, \mathcal{J}_{n}\right\}$ assembles sets $\mathcal{J}_{i}$ that recollect individual features of a critical point $c_{i}$ resting in the Julia set. In each case the size of the set equals the local degree at the said point. We remark once and for all that the marking may not be unique. Also notice that half the marking finishes up empty if there are no critical points in the Fatou or in the Julia set to work with.

Construction of $\mathcal{F}_{k}$. We first consider the case where the Fatou critical point $\omega$ is periodic. Let $\omega \mapsto f(\omega) \mapsto \cdots \mapsto f^{\circ n}(\omega)=\omega$ be a critical cycle of period $n$ and total degree $m>1$. We construct the associated set $\mathcal{F}_{k}$ for every critical element $\omega_{k}$ in the cycle simultaneously. Denote by $d_{\omega}$ the local degree of $f$ at $\omega_{k}$. Pick any periodic point $p_{\omega} \in \partial U(\omega)$ of period dividing $n$-which is not critical since it is periodic and belongs to the Julia set - and consider the left supporting ray $R_{\theta}$ for this component at $p_{\omega}$. This choice, in a natural way, determines periodic supporting rays along the Fatou components in the cycle. Moreover, they are all of period $n$. Given the supporting periodic ray $R_{\theta}$, we track down the $d_{\omega}$ supporting rays of $U(\omega)$ that are inverse images of $f\left(R_{\theta}\right)=R_{d \theta}$. The set of arguments of these rays is $\mathcal{F}_{k}$. Keeping in mind that a preferred periodic supporting ray has already been chosen, we repeat the procedure for all critical points in the cycle. When the cycle has total degree $m$, there are $m-1$ different ways to accomplish the selection. If $\mathcal{F}_{k}$ is the set subordinated to the periodic critical point $\omega_{k}$, there is one argument in $\mathcal{F}_{k}$ which is periodic (namely $\theta$ as above), the so called preferred supporting argument associated with $\omega_{k}$. By definition, the periods of $\omega_{k}$ and of the preferred supporting argument are the same.

Now we consider the case of a nonperiodic Fatou critical point $\omega_{k}$ of degree $d_{\omega}$. There is a minimal $n>0$ for which $\omega^{\prime}=f^{\circ n}(\omega)$ is also critical. Recursively we assume that $\omega^{\prime}$ is marked and has an associated preferred supporting ray $R_{\theta}$ (at the beginning only periodic critical elements have). Then $\left(f^{\circ n}\right)^{-1}\left(R_{\theta}\right)$ contains many rays, but only $d_{\omega}$ among them support this Fatou component $U(\omega)$. Again, the set of arguments of these rays is $\mathcal{F}_{k}$. We pick one, and call it the preferred supporting argument associated with $\omega$. We continue this process for all Fatou critical points until exhausted.

Construction of $\mathcal{J}_{i}$. Given a Julia critical point $c$ of degree $d_{c}>1$, we proceed according to whether its forward orbit is critical point free or not. If the forward orbit of $c$ contains no further critical point, then for some $\theta$, usually nonunique, the ray $R_{\theta}$ lands at the critical value $f(c)$. Now $f^{-1}\left(R_{\theta}\right)$ consists of $d$ different rays, and among them exactly $d_{c}$ land at $c$. As usual, 
define $\mathcal{J}_{c}$ to be the set of arguments of these latter rays. For future reference we choose a preferred one.

Else, the Julia critical point $c$ will reach in $n$ steps another critical point, which we assume to have already associated a preferred ray $R_{\theta}$. In the $n$-fold iterated inverse $\left(f^{\circ n}\right)^{-1}\left(R_{\theta}\right)$ we will discover precisely $d_{c}$ rays landing at $c$. We group these arguments as $\mathcal{J}_{c}$ and select a preferred argument.

Summing up, we see that the construction was made in several steps. First we complete the choice the closest to a periodic orbit, and from there we proceed backwards. At some instances we will face decisions that would affect the subsequent marking. When this happens, we will be performing a hierarchic selection.

The combinatorics of critically marked polynomials. In order to analyze the properties satisfied by $\Theta=(\mathcal{F}, \mathcal{J})$, we introduce combinatorial notation.

A set $\Lambda \subset \mathbb{T}=\mathbb{R} / \mathbb{Z}$ is a degree $d$ preargument set if $d \Lambda=\{d \lambda: \lambda \in \Lambda\}$ is a singleton. For practical reasons it is convenient to assume always that $\Lambda$ contains at least two elements. If all values in $\Lambda$ are rational, we call $\Lambda$ a rational preargument set. As an illustration we single out each individual collection $\mathcal{F}_{\omega}, \mathcal{J}_{c}$ as presented before.

For a family $\Lambda=\left\{\Lambda_{1}, \ldots, \Lambda_{n}\right\}$ of subsets of the circle $\mathbb{T}$, we define the family union as $\Lambda^{\cup}=\bigcup \Lambda_{i}$, and say that $\lambda \in \Lambda^{\cup}$ participates in the family.

Hierarchic families. A family $\Lambda=\left\{\Lambda_{1}, \ldots, \Lambda_{n}\right\}$ is called hierarchic if whenever $\lambda, \lambda^{\prime} \in \Lambda^{\cup}$ and, for some $k$, we have $d^{i} \lambda, d^{j} \lambda^{\prime} \in \Lambda_{k}$ with $i, j>0$, then $d^{i} \lambda=d^{j} \lambda^{\prime}$.

This is like retaining a preferred element $\lambda_{k}$ in each $\Lambda_{k}$. Think of them as gates: as soon as $\lambda \in \Lambda_{l}$ is such that $d^{i} \lambda \in \Lambda_{k}$ for $i>0$, then $d^{i} \lambda$ is the preferred member of $\Lambda_{k}$ chosen before.

Linkage relations. Two subsets $\mathcal{T}, \mathcal{S}$ of the circle are unlinked if they belong to disjoint connected subsets of $\mathbb{T}$, or, equivalently, if $\mathcal{S}$ is contained in one component of $\mathbb{T}-\mathcal{T}$. In particular, these sets are disjoint. If we identify $\mathbb{T}$ with the boundary of the unit disk, an analogous condition is that the convex hulls of $\mathcal{T}$ and of $\mathcal{S}$ are disjoint. When $\mathcal{T}$ and $\mathcal{S}$ are not unlinked, then either $T \cap \mathcal{S} \neq \emptyset$ or there are elements $t_{1}, t_{2} \in \mathcal{T}$ and $s_{1}, s_{2} \in \mathcal{S}$ that can be displayed cyclically as $t_{1}<s_{1}<t_{2}<s_{2}<t_{1}$ (if this is so, they are linked). More generally, a family $\Lambda=\left\{\Lambda_{1}, \ldots, \Lambda_{n}\right\}$ is unlinked if its members are pairwise unlinked. Alternatively, each $\Lambda_{i}$ is contained in a connected component of $\mathbb{T}-\Lambda_{j}$ for all $j \neq i$.

Our definitions are highly motivated by the dynamics of external rays for a polynomial map. For example, if we choose a finite number of different points, and for each we pick the arguments of the rays landing there, then we 
obtain an unlinked family, for otherwise the external rays involved will cross. The same applies for a set of arguments supporting different Fatou components. Nevertheless, when we compare groups of arguments landing at a point against a set of arguments supporting a component, we can anticipate minor problems. Anyhow, it is not difficult to see - because of the consistent way supporting rays are recollected - that rays sharing their landing point are "almost" unlinked to rays supporting a component.

Consider two families $\mathcal{F}=\left\{\mathcal{F}_{1}, \ldots, \mathcal{F}_{n}\right\}$ and $\mathcal{J}=\left\{\mathcal{J}_{1}, \ldots, \mathcal{J}_{m}\right\}$. We say that $\mathcal{J}$ is weakly unlinked to $\mathcal{F}$ on the right if we can choose arbitrarily small $\epsilon>0$ so that the family $\left\{\mathcal{F}_{1}, \ldots, \mathcal{F}_{n}, \mathcal{J}_{1}-\epsilon, \ldots, \mathcal{J}_{m}-\epsilon\right\}$ is unlinked. (Here $\mathcal{J}_{i}-\epsilon=\left\{\lambda-\epsilon: \lambda \in \mathcal{J}_{i}\right\}$.) In particular each family by itself is unlinked. Note that we may even work with empty families.

Formal critical portraits. Consider a pair of families $\mathcal{F}=\left\{\mathcal{F}_{1}, \ldots, \mathcal{F}_{n}\right\}$ and $\mathcal{J}=\left\{\mathcal{J}_{1}, \ldots, \mathcal{J}_{m}\right\}$ of rational degree $d$ preargument sets. We say that $\Theta=(\mathcal{F}, \mathcal{J})$ is a degree $d$ formal critical portrait if the following conditions are fulfilled:

(c1) $d-1=\sum\left(\left|\mathcal{F}_{k}\right|-1\right)+\sum\left(\left|\mathcal{J}_{k}\right|-1\right)$,

(c2) $\mathcal{J}$ is weakly unlinked to $\mathcal{F}$ on the right,

(c3) each family is hierarchic,

(c4) for any $\gamma$ that participates in $\mathcal{F}$, some periodic forward iterate $d^{i} \gamma$ also participates in $\mathcal{F}$,

(c5) no $\theta$ that participates in $\mathcal{J}$ is periodic.

These conditions represent the minimal abstract requirements imposed on a marking. Condition (c1) says that we are choosing the correct number of critical elements. Condition (c2) tells us that the rays and extended rays, once the last ones support a component, determine sectors that do not conflict with each other. Condition (c3) asks for dynamically preferred rays. Condition (c4) favors the fact that arguments in $\mathcal{F}$ are associated with Fatou critical points. Condition ( $\mathrm{c} 5$ ) means that Julia set critical points are non periodic.

Unfortunately, some formal critical portraits are not related to polynomials. To state necessary and sufficient conditions for a marking to arise from a postcritically finite polynomial, we study the partitions of the unit circle they determine.

Given $\Theta=(\mathcal{F}, \mathcal{J})$ as above, we form a partition $\mathcal{P}=\left\{A_{1}, \ldots, A_{d}\right\}$ of the circle minus the finite set of family members $\Theta^{\cup}$ by means of an equivalence relation. We say that two angles $t, s$ not in $\Theta^{\cup}=\mathcal{F}^{\cup} \cup \mathcal{J}^{\cup}$ are unlink equivalent if they belong to the same connected component of $\mathbb{T}-\mathcal{F}_{j}$ and $\mathbb{T}-\mathcal{J}_{i}$ for all possible choices of $i, j$. It is trivial to check that each resulting equivalence class is a finite union of open intervals with total length $1 / d$. 
Each element $A_{i} \in \mathcal{P}$ is a finite union $A_{i}=\bigcup\left(x_{j}, y_{j}\right)$ of open connected intervals. We also define the sets $A_{i}^{+}=\bigcup\left[x_{j}, y_{j}\right)$ and $A_{i}^{-}=\bigcup\left(x_{j}, y_{j}\right]$. This time we get two full partitions $\mathcal{P}^{+}=\left\{A_{1}^{+}, \ldots, A_{d}^{+}\right\}$and $\mathcal{P}^{-}=\left\{A_{1}^{-}, \ldots, A_{d}^{-}\right\}$. As every angle $t$ belongs to only one $A_{k}^{+}$, we recognize in $a_{\Theta}^{+}(t)=A_{k}^{+}$its right addresses. In a similar fashion there is a left addresses $a_{\Theta}^{-}(t)$. We associate to every angle a right symbol sequence $s_{\Theta}^{+}(t)=\left(a_{\Theta}^{+}(t), a_{\Theta}^{+}(d t), a_{\Theta}^{+}\left(d^{2} t\right), \ldots\right)$ and a left one $s_{\Theta}^{-}(t)=\left(a_{\Theta}^{-}(t), a_{\Theta}^{-}(d t), a_{\Theta}^{-}\left(d^{2} t\right), \ldots\right)$. For all but a countable number of arguments (the angles present in the families and their iterated inverses) left and right symbol sequences agree.

Admissible critical portraits. A formal critical portrait $\Theta=(\mathcal{F}, \mathcal{J})$ becomes admissible if two extra conditions are satisfied.

(c6) Suppose $\gamma$ and $t$ are periodic and share period as well as right symbol sequences (i.e., $\left.s_{\Theta}^{+}(\gamma)=s_{\Theta}^{+}(t)\right)$. If $\gamma$ participates in $\mathcal{F}$, then $\gamma=t$.

(c7) Let $\theta \in \mathcal{J}_{l}$ and $\theta^{\prime} \in \mathcal{J}_{k}$. Take $i \geq 0$. If $s_{\Theta}^{-}\left(d^{i} \theta\right)=s_{\Theta}^{-}\left(\theta^{\prime}\right)$ then $d^{i} \theta=\theta^{\prime}$.

Proposition 14.5. If $(f, \Theta)$ is a postcritically finite marked polynomial, then $\Theta$ is an admissible critical portrait.

A brief comment regarding the meaning of these two new conditions is in order. As is usual in this kind of situations, angles sharing a symbol sequence are supposed to land at the same point. Under this proviso, condition (c6) explains why the periodic arguments in $\mathcal{F}^{\cup}$, and not others, support the corresponding component. On the other hand, condition (c7) reinforces the idea that different elements of $\mathcal{J}$ are associated with different critical points, chosen, of course, according to a hierarchic scheme.

Now we are ready to state a structural result for postcritically finite marked polynomials.

TheOREM 14.6. Let $\Theta=(\mathcal{F}, \mathcal{J})$ be a degree $d$ admissible critical portrait. Then there is a unique monic centered postcritically finite polynomial $f$ with marking $(f, \Theta)$.

It is imperative to mention that conditions $(\mathrm{c} 1)-(\mathrm{c} 7)$ represent a finite amount of information to be tested. The next lemmas are important.

LEMMA 14.7. If $\theta$ and $\theta^{\prime}$ have the same periodic left or right symbol sequence, then $\theta$ and $\theta^{\prime}$ are both periodic and share the same period.

LEMMA 14.8. The symbol sequence $s_{\Theta}^{-}(\theta)$ is periodic of period $m$ if and only if the landing point of the ray $R_{\theta}$ has period $m$.

The last question we ask is what kind of information about the Julia set can be inferred by studying the combinatorics. For example, we wonder if we can anticipate if two rays land at the same point by looking at their 
arguments. In fact, left symbol sequences convey the information needed to decide whether or not two rays land at the same point. This is done as follows.

DeFinition 14.9. The landing relation generated by an admissible critical portrait $\Theta=(\mathcal{F}, \mathcal{J})$, denoted $\sim_{l}$, is the smallest equivalence relation in $\mathbb{T}$ such that if one of the following two conditions hold, then $s$ and $t$ are $\sim_{l}$-related:

(11) $s_{\Theta}^{-}(s)=s_{\Theta}^{-}(t)$,

(12) there is $j$ such that $a_{\Theta}^{-}\left(d^{i} s\right)=a_{\Theta}^{-}\left(d^{i} t\right)$ for all $i<j$ and $\left\{d^{j} s, d^{j} t\right\}$ $\subset \mathcal{J}_{k}$ for some $k$.

It is important to learn how to recognize by inspection if two arguments are $\sim_{l}$-related. An irrational or a periodic argument can only be related to arguments with identical left symbol sequence. In fact, those values and their forward orbits are unfit to belong to $\mathcal{J}_{k}$ because $\mathcal{J}^{\cup}$ contains only strictly preperiodic elements. Therefore, all possible exceptions are to be blamed to the fact that future iterates share a symbol sequence with a marked "Julia" element.

For example, conditions (c7) and (c3) combined guarantee that whenever $\theta_{k} \in \mathcal{J}_{k}(k=0,1)$, then we can have $\theta_{0} \sim_{l} \theta_{1}$ if and only if $\mathcal{J}_{0}=\mathcal{J}_{1}$.

In practice, two arguments $\theta_{0}, \theta_{n}$ end up $\sim_{l}$-related by means of a finite sequence $\theta_{0}, \theta_{1}, \ldots, \theta_{n}$ where one argument is related to the next via condition (11) or (12) above. It is also true that only a finite number of arguments can appear in an equivalence class.

The next result allows us to use induction when needed.

Lemma 14.10. Let $a_{\Theta}^{-}(\theta)=a_{\Theta}^{-}\left(\theta^{\prime}\right)$. Then $\theta \sim_{l} \theta^{\prime}$ if and only if $d \theta \sim_{l} d \theta^{\prime}$.

This equivalence relation captures the essence of two rays landing together, as our last proposition indicates.

Proposition 14.11. Let $(f, \Theta)$ be a critically marked polynomial. Then the rays $R_{\theta}, R_{\theta^{\prime}}$ land at the same point if and only if $\theta \sim_{l} \theta^{\prime}$.

Acknowledgments. The main ideas of this work comprise part of the author's PhD thesis [P1] at SUNY Stony Brook. We wish to thank John Milnor for his insight and helpful suggestions.

\section{References}

[D] A. Douady, Descriptions of compact sets in $\mathbb{C}$, in: Topological Methods in Modern Mathematics: A Symposium in Honor of John Milnor's Sixtieth Birthday, Publish or Perish, 1993, 429-465.

[DH] A. Douady et J. Hubbard, Étude dynamique des polynômes complexes, I, II, Publ. Math. Orsay 1984-1985. 
[L] P. Lavaurs, Thèse, Univ. de Paris-Sud, Centre d'Orsay, 1989.

[M] J. Milnor, Dynamics in One Complex Variable, Introductory Lectures, Vieweg, 1999.

[P1] A. Poirier, On postcritically finite polynomials, PhD Thesis, SUNY Stony Brook, 1993.

[P2] -, On postcritically finite polynomials, Part two: Hubbard trees, Preprint IMS Stony Brook 7/1993.

[P3] —, Critical portraits for postcritically finite polynomials, Fund. Math. 203 (2009), 107-163.

Alfredo Poirier

Departmento de Ciencias

Sección Matemáticas

Pontificia Universidad Católica del Perú

Apartado 1761

Lima 100, Perú

E-mail: apoirie@pucp.edu.pe

Received 20 April 2009;

in revised form 21 April 2010 